\title{
Equations for two-phase flows: a primer
}

\author{
Andrea Dziubek
}

June 2, 2018

\section{Contents}

1 Introduction

2 Geometry of a moving interface 4

2.1 Curvilinear coordinate systems . . . . . . . . . . . . . . . . 4

2.2 Mean curvature . . . . . . . . . . . . . . . . . . 7

2.3 Implicit parameterized surface . . . . . . . . . . . . . 7

3 Kinematics of bulk fluids and of the moving interface $\quad 10$

3.1 Kinematics of a material volume . . . . . . . . . . . . . . . 10

3.2 Reynolds transport theorem for a material volume with an interface . . . . . . 13

3.3 Kinematics of a moving interface and velocities . . . . . . . . . . . 15

3.4 Reynolds transport theorem and divergence theorem for a surface . . . . . . . . 19

4 Generic model equations for two-phase flows with surface tension 21

4.1 Balance equations for bulk fluids . . . . . . . . . . . . . . . . 21

4.2 Jump conditions at an interface between two fluids . . . . . . . . . . . . . 23

4.3 Balance equation and jump condition including surface tension . . . . . . . . 24

5 Model equations with phase change and surface tension 26

5.1 Mass, momentum and energy equation . . . . . . . . . . . . . . . . . . .

5.2 Mass, momentum and energy jump conditions . . . . . . . . . . . . . . . . . . . . . . . . . . . .

5.3 Summary of the model equations . . . . . . . . . . . . . . . 30 


\section{Introduction}

The subject of Physics of fluids and heat transfer have been well established during the last century, and has been intensively studied for a wide range of hydrodynamical problems. Many current efficient computational fluid dynamics (CFD) software packages offer great flexibility in geometry and material properties. However, two-phase flow problems with moving boundaries still present a major challenge to the current state of computational fluid dynamics.

Although an interface is a three-dimensional region with a thickness of the order of molecular diameters it is conveniently modeled as a two dimensional surface (Cosserat surface). Surface tension, being a geometrical property of a two dimensional surface, is proportional to the curvature of the interface. It is also a material property of the two adjoined materials, thus it is an intrinsic property of the surface. In order to include surface tension properly into the modeling we need to formulate an additional balance equation for the surface and add it to the volume balance equation.

Interfacial dynamics for Newtonian surface fluids was first described by Scriven in [Sc] using tensor notation. However, tensor notation is not part of a typical engineering education. Also, there is scarce literature on interface balance equations covering surface tension, most use a source term instead.

The main goal of these notes is to give a review of the equations for two phase flow problems with an interface between the two phases in a self-contained way, and, in particular, to properly include surface tension into the interface balance equations.

Balance equations at an interface involve geometrical quantities, such as normal and tangential vectors on the interface and mean curvature of the interface. We recall them in section 2 , For the balance equations and the jump conditions we need Reynolds' transport theorems for a material body and for a material body with an internal interface. We review them in section 3.1 and 3.2. The kinematics of a moving surface are described in section 3.3 .

In section 4.1 and 4.2 we recall balance equations for single phase problems and ordinary jump conditions for an interface, where the adjacent phases are incompressible Newtonian fluids. Taking surface tension into account increases the complexity. The normal and tangential vectors on the interface can be derived by a simple geometric demonstration. However, to describe mean curvature (in a physical, intuitive way) use of tensor notation becomes inevitable. The jump condition from section 4.2 does not cover surface tension.

Surface tension is proportional to the curvature of the interface, being a geometrical property of a surface. It is also a material property of the two related materials, thus it is an 
intrinsic property of the surface. In order to include surface tension properly into a jump condition we need to formulate an additional balance equation for the surface and add it to the volume balance equation. This is done in section 4.3. In return it involves kinematic relations of the interface and the Reynolds transport theorem for surfaces. They are given in section 3.3 and 3.4 .

Finally, in section 5, the generic balance equations are applied to mass momentum and energy and common simplifications and boundary conditions are discussed.

Preliminaries In this text we use Einstein summation convention, which states a repeated index implies a sum over all possible values for that index. When the index takes only the values 1,2, Greek letters are used and when the index takes the values 1,2,3, Latin letters are used. A vector in a general curvilinear coordinate system can be referred to the standard basis $\left\{\boldsymbol{e}_{i}\right\}$ or to the dual basis $\left\{\boldsymbol{e}^{i}\right\}$ and we can write $\boldsymbol{v}=v^{i} \boldsymbol{e}_{i}$ or $\boldsymbol{v}=v_{i} \boldsymbol{e}^{i}$. The $v^{i}$ are called contravariant components and the $v_{i}$ are called covariant components of the vector $\boldsymbol{v}$. The dual basis is defined by $\boldsymbol{e}_{i} \cdot \boldsymbol{e}^{j}=\delta_{i}{ }^{j}$. In a Cartesian coordinate system there is no difference between standard and dual basis and the contravariant and covariant coordinates of a vector are the same. In a curvilinear coordinate system the basis vectors carry a part of the length information of a vector.

The tensor (outer) product is defined by its action

$$
(\boldsymbol{u} \otimes \boldsymbol{v}) \boldsymbol{w}=\boldsymbol{u}(\boldsymbol{v} \cdot \boldsymbol{w}) \quad \text { or } \quad \boldsymbol{u}(\boldsymbol{v} \otimes \boldsymbol{w})=(\boldsymbol{u} \cdot \boldsymbol{v}) \boldsymbol{w} .
$$

Computing the outer products of the base vectors a second order tensor can be represented as $\boldsymbol{T}=T^{i j} \boldsymbol{e}_{i} \otimes \boldsymbol{e}_{j}=T^{i}{ }_{j} \boldsymbol{e}_{i} \otimes \boldsymbol{e}^{j}=T_{i}{ }^{j} \boldsymbol{e}^{i} \otimes \boldsymbol{e}_{j}=T_{i j} \boldsymbol{e}^{i} \otimes \boldsymbol{e}^{j}$. The scalar (dot) product between two tensors is defined as

$$
\left(\boldsymbol{v}_{1} \otimes \boldsymbol{v}_{2}\right) \cdot\left(\boldsymbol{w}_{1} \otimes \boldsymbol{w}_{2}\right)=\left(\boldsymbol{v}_{1} \cdot \boldsymbol{w}_{1}\right)\left(\boldsymbol{v}_{2} \cdot \boldsymbol{w}_{2}\right) .
$$

The gradient of a vector is defined as

$$
\boldsymbol{\nabla} \boldsymbol{v}=\boldsymbol{e}_{i} \frac{\partial}{\partial x^{i}} \otimes \boldsymbol{v}
$$

and the divergence of a second order tensor is defined as

$$
\boldsymbol{\nabla} \cdot \boldsymbol{S}=\boldsymbol{e}_{k} \frac{\partial}{\partial x^{k}}\left(S^{i j} \boldsymbol{e}_{i} \otimes \boldsymbol{e}_{j}\right)=\left(\boldsymbol{e}_{k} \frac{\partial}{\partial x^{k}} \cdot S^{i j} \boldsymbol{e}_{i}\right) \boldsymbol{e}_{j}=\frac{\partial S^{i j}}{\partial x^{i}} \boldsymbol{e}_{j}
$$

Alternatively one could first take the derivative and then compute the dot or tensor product, i.e. $\boldsymbol{\nabla} \boldsymbol{v}=\frac{\partial \boldsymbol{v}}{\partial x^{k}} \otimes \boldsymbol{e}_{k}$ or $\boldsymbol{\nabla} \cdot \boldsymbol{S}=\frac{\partial \boldsymbol{S}}{\partial x^{k}} \cdot \boldsymbol{e}_{k}$. Note that both definitions are possible and are used in the literature. Our definition is more common in fluid dynamics, the later definition is more common in elasticity. 


\section{Geometry of a moving interface}

In this section we summarize the geometrical properties of the moving interface between two phases. First, the formulas for normal and tangential vectors, mean curvature and interface velocity are given. Then, using an implicit representation of the surface, the geometrical properties of two phases at the interface are computed. Two formulas to compute the normal vector and two formulas to compute the mean curvature are given.

The material in this section is mainly based on [Ed], $[\mathrm{Ar}],[\mathrm{TrTo}$ and $[\mathrm{Er}$. They present a formulation of classical continuum physics using tensor calculus. For an introduction in tensor calculus we refer to [Scha]. S107], S199] and [De present transport phenomena (including interphase transport phenomena) using moderate tensor notation. [Kü] is a differential geometry book written more for mathematicians, Op has an easier notation but lack some derivations. For advanced calculus we refer to $\mathrm{Ap}]$ and $[\mathrm{Ka}]$.

\subsection{Curvilinear coordinate systems}

A two-dimensional surface can best be analyzed by covering the surface with a grid, see figure 1. The grid is obtained by the curves where $u^{1}$ and $u^{2}$ are constant. The position of a point on the surface can be given intrinsically in terms of the two curvilinear surface coordinates (or parameters) $u^{1}$ and $u^{2}$, or extrinsically by a position vector to the point. This defines a curvilinear co-

ordinate system which is not orthogonal in general.

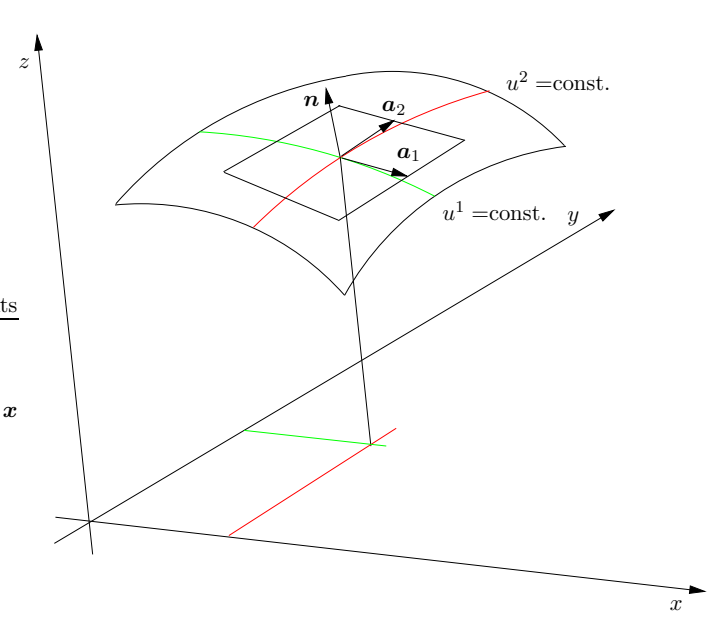

Figure 1: Tangent space on a surface

Tangential and normal vectors If the interface between the two phases is not stationary the position vector to a point on the interface is given in cartesian coordinates as

$$
\boldsymbol{x}\left(u^{1}, u^{2}, t\right)=x^{1}\left(u^{1}, u^{2}, t\right) \boldsymbol{e}_{1}+x^{2}\left(u^{1}, u^{2}, t\right) \boldsymbol{e}_{2}+x^{3}\left(u^{1}, u^{2}, t\right) \boldsymbol{e}_{3},
$$

or in index notation as

$$
\boldsymbol{x}\left(u^{\alpha}, t\right)=x^{i}\left(u^{\alpha}, t\right) \boldsymbol{e}_{i},
$$


where $u^{\alpha}=u^{1}, u^{2}$.

A Taylor series expansion of $\boldsymbol{x}$ in the surface variables $u^{\alpha}$ up to the linear term yields the total derivative

$$
\boldsymbol{x}\left(u^{\alpha}+\mathrm{d} u^{\alpha}\right)-\boldsymbol{x}\left(u^{\alpha}\right)=\mathrm{d} \boldsymbol{x}=\frac{\partial \boldsymbol{x}}{\partial u^{\alpha}} \mathrm{d} u^{\alpha} .
$$

Along $u^{1}$-curves, $u^{2}=$ constant $\left(\mathrm{d} u^{2}=0\right), \frac{\partial \boldsymbol{x}}{\partial u^{1}}$ defines the tangent vector along this curves; similarly $\frac{\partial \boldsymbol{x}}{\partial u^{2}}$ defines the tangent vector along the $u^{2}$ curve. The derivatives with respect to the curvilinear coordinates $u^{\alpha}$ are called covariant derivatives. The covariant derivatives of a position vector

$$
\boldsymbol{a}_{\alpha}=\frac{\partial \boldsymbol{x}}{\partial u^{\alpha}}=\boldsymbol{e}_{i} \frac{\partial x^{i}}{\partial u^{\alpha}}
$$

form the base vectors of a local surface coordinate system. In terms of the covariant base vectors the surface metric tensor is defined as

$$
a_{\alpha \beta}=\boldsymbol{a}_{\alpha} \cdot \boldsymbol{a}_{\beta}
$$

The metric tensor is also called the first fundamental form. The local unit normal vector at a point $\left(u^{1}, u^{2}\right)$ normal to the surface is defined by

$$
\boldsymbol{n}=\frac{\boldsymbol{a}_{1} \times \boldsymbol{a}_{2}}{\left|\boldsymbol{a}_{1} \times \boldsymbol{a}_{2}\right|} .
$$

Dual basis Another set of base vectors $\left\{\boldsymbol{a}^{\beta}\right\}$, is defined by the surface Kronecker delta,

$$
\boldsymbol{a}_{\alpha} \cdot \boldsymbol{a}^{\beta}=\delta_{\alpha}^{\beta}
$$

they are called dual base vectors or reciprocal or contravariant base vectors respectively. This condition orthogonality relation defines a vector $\boldsymbol{a}^{1}$ that lies in the plane formed by the vectors $\boldsymbol{a}_{1}, \boldsymbol{a}_{2}$, is perpendicular to $\boldsymbol{a}_{2}$, forms an acute angle with $\boldsymbol{a}_{1}$. This is also the definition of the gradient $\nabla u^{1}$ of the surface coordinate, which is perpendicular to the level surface defined by $u^{1}\left(x^{1}, x^{2}, x^{3}, t\right)=$ constant. Similarly the orthogonality relation defines the vector $\boldsymbol{a}^{2}$. Then the dual or contravariant base vectors are given by

$$
\boldsymbol{a}^{\alpha}=\nabla u^{\alpha}=\boldsymbol{e}_{i} \frac{\partial u^{\alpha}}{\partial x^{i}}
$$

With the contravariant base vectors the covariant surface metric tensor is

$$
a^{\alpha \beta}=\boldsymbol{a}^{\alpha} \cdot \boldsymbol{a}^{\beta} .
$$


However, often the dual basis is more conveniently calculated by means of the local unit normal vector

$$
\boldsymbol{a}^{1}=\frac{\boldsymbol{a}_{2} \times \boldsymbol{n}}{\left[\boldsymbol{a}_{1}, \boldsymbol{a}_{2}, \boldsymbol{n}\right]}, \quad \boldsymbol{a}^{2}=\frac{\boldsymbol{n} \times \boldsymbol{a}_{1}}{\left[\boldsymbol{a}_{1}, \boldsymbol{a}_{2}, \boldsymbol{n}\right]} .
$$

$\left[\boldsymbol{a}_{1}, \boldsymbol{a}_{2}, \boldsymbol{n}\right]=\left[\boldsymbol{a}_{1} \times \boldsymbol{a}_{2}\right] \cdot \boldsymbol{n}$ is the scalar triple product.

Orthogonal curvilinear coordinate systems If the base vectors of a curvilinear coordinate system are mutually orthogonal $\left(\boldsymbol{a}_{1} \cdot \boldsymbol{a}_{2}=0\right)$, then the covariant and contravariant metric tensor reduce simply to

$$
a_{\alpha \beta}=\left[\begin{array}{cc}
a_{11} & 0 \\
0 & a_{22}
\end{array}\right], \quad a^{\alpha \beta}=\left[\begin{array}{cc}
a^{11} & 0 \\
0 & a^{22}
\end{array}\right] .
$$

For such orthogonal systems the normalized surface vectors are called self reciprocal, in the sense that $\frac{a_{1}}{\sqrt{a_{11}}}=\frac{\boldsymbol{a}^{1}}{\sqrt{a^{11}}}$ and $\frac{\boldsymbol{a}_{2}}{\sqrt{a_{22}}}=\frac{\boldsymbol{a}^{2}}{\sqrt{a^{22}}}$. It is convenient to introduce unit vectors

$$
\boldsymbol{e}_{1}=\frac{\boldsymbol{a}_{1}}{\sqrt{a_{11}}}, \quad \boldsymbol{e}_{2}=\frac{\boldsymbol{a}_{2}}{\sqrt{a_{22}}}
$$

Surface gradient The identity tensor is defined by

$$
\boldsymbol{I}=\boldsymbol{a}^{1} \otimes \boldsymbol{a}_{1}+\boldsymbol{a}^{2} \otimes \boldsymbol{a}_{2}+\boldsymbol{n} \otimes \boldsymbol{n} .
$$

It possesses the property $\boldsymbol{I} \boldsymbol{x}=\boldsymbol{x}$ for any $\boldsymbol{x}$. This relation is also called orthogonality relation. By subtracting the part related to the normal vector from $\boldsymbol{I}$ the surface identity tensor is defined as

$$
\boldsymbol{I}_{S}=\boldsymbol{I}-\boldsymbol{n} \otimes \boldsymbol{n}=\boldsymbol{a}^{\alpha} \otimes \boldsymbol{a}_{\alpha} .
$$

Similarly the surface gradient is defined by the projection in normal direction subtracted from the gradient

$$
\boldsymbol{\nabla}_{S}=\boldsymbol{I}_{S} \boldsymbol{\nabla}=(\boldsymbol{I}-\boldsymbol{n} \otimes \boldsymbol{n}) \boldsymbol{\nabla}=\boldsymbol{\nabla}-(\boldsymbol{n} \otimes \boldsymbol{n}) \boldsymbol{\nabla} .
$$

By this we get

$$
\begin{aligned}
\nabla_{S} & =\boldsymbol{a}^{\alpha} \otimes \boldsymbol{a}_{\alpha}\left(\boldsymbol{e}_{j} \frac{\partial}{\partial x^{j}}\right)=\boldsymbol{a}^{\alpha}\left(\boldsymbol{a}_{\alpha} \cdot \boldsymbol{e}_{j} \frac{\partial x^{j}}{\partial u^{\alpha}}\right)=\boldsymbol{a}^{\alpha}\left(\boldsymbol{e}_{i} \frac{\partial x^{i}}{\partial u^{\alpha}} \cdot \boldsymbol{e}_{j} \frac{\partial}{\partial x^{j}}\right) \\
& =\boldsymbol{a}^{\alpha} \frac{\partial}{\partial u^{\alpha}} .
\end{aligned}
$$




\subsection{Mean curvature}

The mean curvature is proportional to the rate of change of the local normal vector with respect to the surface coordinates.

$$
H=-\frac{1}{2} \nabla_{S} \cdot \boldsymbol{n}=-\frac{1}{2}\left(\boldsymbol{a}^{\alpha} \frac{\partial}{\partial u^{\alpha}}\right) \cdot \boldsymbol{n} .
$$

\subsection{Implicit parameterized surface}

To compute the normal and tangential vectors and the mean curvature of the moving interface it is necessary to choose a parametrization of the interface. For example, if a vertical tube is assumed where a thin film is flowing down along the inner walls (without waves), then the problem has rotational symmetry and the interface between the liquid and the gas can be parameterized with the surface coordinates $u^{1}=z$ and $u^{2}=\vartheta$. To assume an implicit parameterized surface is more general.

Normal and tangential vectors of an implicit parametrized surface Every moving surface can be locally described by a real-valued function of two variables and time

$$
z=h(u, v, t) \quad \text { or implicitly } \quad F(u, v, z, t)=z-h(u, v, t)=0 .
$$

For convenience we write $u^{1}=u, u^{2}=v$. By this parametrization the position vector to any point on the surface becomes

$$
\boldsymbol{x}(u, v, t)=u \boldsymbol{e}_{1}+v \boldsymbol{e}_{2}+h(u, v, t) \boldsymbol{e}_{3} .
$$

The tangential vectors are given by the covariant base vectors (31) as

$$
\boldsymbol{a}_{1}=\frac{\partial \boldsymbol{x}}{\partial u}=\left[\begin{array}{c}
1 \\
0 \\
\frac{\partial h}{\partial u}
\end{array}\right], \quad \boldsymbol{a}_{2}=\frac{\partial \boldsymbol{x}}{\partial v}=\left[\begin{array}{c}
0 \\
1 \\
\frac{\partial h}{\partial v}
\end{array}\right] .
$$

The local unit normal vector is the cross product of the tangent vectors, scaled by its length. Alternatively, if the surface is given by $z=h(u, v, t)$, the unit normal vector can be obtained from the gradient of the implicit function $F(u, v, z, t)=0$. Expanding $F(u, v, z, t)$ in a Taylor series up to the linear term for the variables $u, v, z$ leads to the total derivative

$$
\mathrm{d} F=\frac{\partial F}{\partial u} \mathrm{~d} u+\frac{\partial F}{\partial v} \mathrm{~d} v+\frac{\partial F}{\partial z} \mathrm{~d} z
$$


which is zero because $F=0$. Writing the total derivative as $\boldsymbol{\nabla} F \cdot \mathrm{d} \boldsymbol{u}=0$ where $\mathrm{d} \boldsymbol{u}=$ $[\mathrm{d} u, \mathrm{~d} v, \mathrm{~d} z]$ shows that the gradient $\nabla F$ is perpendicular to the level surface defined by $F(u, v, z, t)=0$. The local unit normal vector reads then

$$
\boldsymbol{n}=\frac{\boldsymbol{\nabla} F}{|\boldsymbol{\nabla} F|}=\left[\begin{array}{c}
-\frac{\partial h}{\partial u} \\
-\frac{\partial h}{\partial v} \\
1
\end{array}\right] \frac{1}{\sqrt{\left(\frac{\partial h}{\partial u}\right)^{2}+\left(\frac{\partial h}{\partial v}\right)^{2}+1}} .
$$

Mean curvature of an implicit parametrized surface The mean curvature is given by (13) as

$$
H=-\frac{1}{2}\left(\boldsymbol{a}^{1} \cdot \frac{\partial \boldsymbol{n}}{\partial u}+\boldsymbol{a}^{2} \cdot \frac{\partial \boldsymbol{n}}{\partial v}\right)
$$

Alternatively the mean curvature is often computed more conveniently by means of the first and second fundamental form as explained below. For the implicit surface parametrization the covariant metric tensor, or first fundamental form (44), reads in matrix form

$$
a_{\alpha \beta}=\left[\begin{array}{ll}
\boldsymbol{a}_{1} \cdot \boldsymbol{a}_{1} & \boldsymbol{a}_{1} \cdot \boldsymbol{a}_{2} \\
\boldsymbol{a}_{2} \cdot \boldsymbol{a}_{1} & \boldsymbol{a}_{2} \cdot \boldsymbol{a}_{2}
\end{array}\right]=\left[\begin{array}{cc}
1+\left(\frac{\partial h}{\partial u}\right)^{2} & \frac{\partial h}{\partial u} \frac{\partial h}{\partial v} \\
\frac{\partial h}{\partial v} \frac{\partial h}{\partial u} & 1+\left(\frac{\partial h}{\partial v}\right)^{2}
\end{array}\right] .
$$

The contravariant metric tensor $a^{\alpha \gamma}$ is defined as the inverse of the covariant metric tensor, $a^{\alpha \gamma} a_{\gamma \beta}=\delta_{\beta}^{\alpha}$. An element of the inverse of a matrix is given by the transpose of the cofactor matrix (denoted by a tilde), divided by the determinant of the matrix. This yields for a $(2 \times 2)$ matrix

$$
a^{\alpha \beta}=\left(a_{\alpha \beta}\right)^{-1}=\frac{\tilde{a}_{\alpha \beta}}{\operatorname{det} a_{\alpha \beta}}=\frac{1}{a_{11} a_{22}-a_{12} a_{21}}\left[\begin{array}{cc}
a_{22} & -a_{12} \\
-a_{21} & a_{11}
\end{array}\right],
$$

and for the implicit surface parametrization

$$
a^{\alpha \beta}=\frac{1}{1+\left(\frac{\partial h}{\partial u}\right)^{2}+\left(\frac{\partial h}{\partial v}\right)^{2}}\left[\begin{array}{cc}
1+\left(\frac{\partial h}{\partial v}\right)^{2} & -\frac{\partial h}{\partial u} \frac{\partial h}{\partial v} \\
-\frac{\partial h}{\partial u} \frac{\partial h}{\partial v} & 1+\left(\frac{\partial h}{\partial u}\right)^{2}
\end{array}\right] .
$$

Both metric tensors are evidently symmetric. They are also positive definite 1

Next, the second fundamental form is defined as

$$
b_{\alpha \beta}=\frac{\partial \boldsymbol{a}_{\alpha}}{\partial u^{\beta}} \cdot \boldsymbol{n} \quad \text { or alternatively } \quad b_{\alpha \beta}=-\boldsymbol{a}_{\alpha} \cdot \frac{\partial \boldsymbol{n}}{\partial u_{\beta}} .
$$

\footnotetext{
${ }^{1}$ The condition that a matrix is positive definite is that all upper left determinants are positive, $a^{11}=$ $1+\left(\frac{\partial h}{\partial u}\right)^{2}>0$ and $a^{11} a^{22}-\left(a^{12}\right)^{2}=\left(1+\left(\frac{\partial h}{\partial u}\right)^{2}\right)\left(1+\left(\frac{\partial h}{\partial v}\right)^{2}\right)-\frac{\partial h}{\partial u} \frac{\partial h}{\partial v}=1+\left(\frac{\partial h}{\partial u}\right)^{2}+\left(\frac{\partial h}{\partial u}\right)^{2}+\frac{\partial h}{\partial u} \frac{\partial h}{\partial v}-\frac{\partial h}{\partial u} \frac{\partial h}{\partial v}>0$.
} 
The first equation of (16) yields for our parametrization

$$
b_{\alpha \beta}=\frac{\partial \boldsymbol{a}_{\alpha}}{\partial \beta} \cdot \boldsymbol{n}=-\left[\begin{array}{cc}
\frac{\partial \boldsymbol{a}_{1}}{\partial u} \cdot \boldsymbol{n} & \frac{\partial \boldsymbol{a}_{1}}{\partial v} \cdot \boldsymbol{n} \\
\frac{\partial \boldsymbol{a}_{2}}{\partial u} \cdot \boldsymbol{n} & \frac{\partial \boldsymbol{a}_{2}}{\partial v} \cdot \boldsymbol{n}
\end{array}\right]=\frac{1}{1+\left(\frac{\partial h}{\partial u}\right)^{2}+\left(\frac{\partial h}{\partial v}\right)^{2}}\left[\begin{array}{cc}
\frac{\partial^{2} h}{\partial u^{2}} & \frac{\partial^{2} h}{\partial u \partial v} \\
\frac{\partial^{2} h}{\partial v \partial u} & \frac{\partial^{2} h}{\partial v^{2}}
\end{array}\right] .
$$

The second fundamental form is also symmetric but not necessarily positive definite. From the first and second fundamental form the shape operator or Weingarten map is defined

$$
L=b_{\alpha \gamma} a^{\gamma \beta}=\left[\begin{array}{ll}
b_{11} & b_{12} \\
b_{21} & b_{22}
\end{array}\right] \frac{1}{\operatorname{det} a_{\alpha \beta}}\left[\begin{array}{cc}
a_{22} & -a_{12} \\
-a_{21} & a_{11}
\end{array}\right] \text {. }
$$

It becomes for the implicit surface parametrization

$$
L=\frac{1}{\sqrt{o}}\left[\begin{array}{ll}
\frac{\partial^{2} h}{\partial u^{2}}\left(1+\left(\frac{\partial h}{\partial v}\right)^{2}\right)-\frac{\partial^{2} h}{\partial u \partial v} \frac{\partial h}{\partial u} \frac{\partial h}{\partial v} & \frac{\partial^{2} h}{\partial v \partial u}\left(1+\left(\frac{\partial h}{\partial u}\right)^{2}\right)-\frac{\partial^{2} h}{\partial u^{2}} \frac{\partial h}{\partial u} \frac{\partial h}{\partial v} \\
\frac{\partial^{2} h}{\partial u \partial v}\left(1+\left(\frac{\partial h}{\partial v}\right)^{2}\right)-\frac{\partial^{2} h}{\partial v^{2}} \frac{\partial h}{\partial u} \frac{\partial h}{\partial v} & \frac{\partial^{2} h}{\partial v^{2}}\left(1+\left(\frac{\partial h}{\partial u}\right)^{2}\right)-\frac{\partial^{2} h}{\partial u \partial v} \frac{\partial h}{\partial u} \frac{\partial h}{\partial v}
\end{array}\right],
$$

with $\sqrt{o}=\sqrt{1+\left(\frac{\partial h}{\partial u}\right)^{2}+\left(\frac{\partial h}{\partial v}\right)^{2}}$. $L$ is the product of a symmetric positive definite matrix and a symmetric matrix and the eigenvalues of such a product are all real.

The two eigenvalues $\kappa_{1}$ and $\kappa_{2}$ of $L$ are called principal curvatures. The mean curvature and the Gauß curvature $K$ are defined by

$$
\begin{aligned}
H & =\frac{1}{2} \operatorname{trace} L=\frac{1}{2} b_{\alpha \beta} a^{\alpha \beta}=\kappa_{1}+\kappa_{2}, \\
K & =\operatorname{det} L=\frac{\operatorname{det} b_{\alpha \beta}}{\operatorname{det} a_{\alpha \beta}}=\kappa_{1} \kappa_{2} .
\end{aligned}
$$

So that finally the mean curvature becomes for our parametrization

$$
H=\frac{1}{2}\left(\frac{\frac{\partial^{2} h}{\partial u^{2}}\left(1+\left(\frac{\partial h}{\partial v}\right)^{2}\right)-2 \frac{\partial^{2} h}{\partial u \partial v} \frac{\partial h}{\partial u} \frac{\partial h}{\partial v}+\frac{\partial^{2} h}{\partial v^{2}}\left(1+\left(\frac{\partial h}{\partial u}\right)^{2}\right)}{\left.\left(\frac{\partial h}{\partial u}\right)^{2}+\left(\frac{\partial h}{\partial v}\right)^{2}+1\right)^{\frac{3}{2}}}\right) .
$$

From the two equations for the mean curvature, (18) involves only the covariant base vectors and the derivatives of the covariant base vectors, whereas (13) involves also the contravariant base vectors and their derivatives, so that is often easier to use(18) to compute the mean curvature, especially in the case of orthogonal coordinate systems. However, the definition of the mean curvature with (13) is more physically intuitive.

For the computation of the mean curvature with (18) only the covariant base vectors and the derivatives of the covariant base vectors need to be computed. If (13) is used to compute the mean curvature, the contravariant base vectors and their derivatives will also need to be computed. Using the shape operator to compute the mean curvature often simplifies the computations, especially in the case of orthogonal coordinate systems. 


\section{Kinematics of bulk fluids and of the moving interface}

In this section we present kinematical relations that are necessary to formulate the balance equations at an interface between two phases. Experiments show that a fluid interface is in fact a three-dimensional region with a thickness on the micro-scale level. Following [Gi], such an interface can be regarded as a two-dimensional dividing surface where the effects of the interface on the adjoining bulk phases are represented by surface excess mass, momentum and energy.

First we give kinematical relations for the bulk fluids, then we consider a material volume with an internal interface. Next we discuss the kinematical relations of the two-dimensional moving interface and in the last section we deal with kinematical relations related to the curvature of the interface.

The first part of this section is mainly based on [Ar], [Scha] and [TrTo]. For the interface related sections see also [S107], [S199] and [Ed].

\subsection{Kinematics of a material volume}

To clarify terminology we first recall some kinematics of bulk fluids and give the Reynolds transport theorem for a material volume.

From the basic assumption of continuum theory, a body consists of infinitely many particles without dimension and no space between them and every particle corresponds to a position in space. A particle is represented at a given initial time, by a position vector $\boldsymbol{\xi}$, as shown in figure 2, The coordinates of $\boldsymbol{\xi}$ are called material coordinates. At another time the same particle is represented by another position vector as a function of the initial position of the particle and time

$$
\boldsymbol{x}=\boldsymbol{x}(\boldsymbol{\xi}, t)
$$

The coordinates of $\boldsymbol{x}$ are called spatial coordinates.

The initial position of the particle is taken as a reference configuration. Equation (21) defines the motion of a particle. Assuming continuous motion and that a particle can not occupy two places at the same time the relation is a one-to-one mapping and we can also write conversely

$$
\boldsymbol{\xi}=\boldsymbol{\xi}(\boldsymbol{x}, t) .
$$


Physical quantities like density, velocity and temperature, which are functions of space and time, are called field variables and they are here denoted by $\varphi$. A field variable can also be given as a function of particle and time. The representation of a field variable as a function of space and time is called spatial (or Euler) representation, that is

$$
\varphi=\varphi(\boldsymbol{x}, t) \quad \text { or } \quad \varphi=\varphi(\boldsymbol{\xi}(\boldsymbol{x}, t), t) .
$$

The representation of a field variable as a function of particle and time is called material (or Lagrange) representation, that is

$$
\varphi=\varphi(\boldsymbol{\xi}, t) \quad \text { or } \quad \varphi=\varphi(\boldsymbol{x}(\boldsymbol{\xi}, t), t) .
$$

Balance equations of mass, momentum and energy are appropriately described in an Eulerian framework.

Material derivative and velocity Field variables are functions of several yariables, so their derivatives are partial derivatives. Partial derivatives wherespatial cooromates are held constant are denoted by $\partial$. Partial derivatives where matexial coordinates arefheld constant we denote with an uppercase D. 2 The partial derivative with respect to time

$$
\frac{\mathrm{D} \varphi}{\mathrm{D} t}=\frac{\partial \varphi(\boldsymbol{\xi}, t)}{\partial t}=\left(\frac{\partial \varphi}{\partial t}\right)_{\boldsymbol{\xi}} .
$$

is called material (or convected) derivative and gives the rate off change which an observer moving with the particle would see. The material derivative of a position vector is the velocity of a given particle

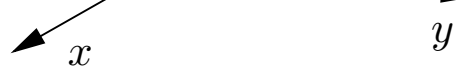

$$
\boldsymbol{v}=\frac{\mathrm{D} \boldsymbol{x}}{\mathrm{D} t} .
$$

Figure 2: Moving particle

Balance equations are given in spatial coordinates. To obtain the material derivative of a field variable $\varphi(\boldsymbol{x}(\boldsymbol{\xi}, t), t)$ in spatial variables the chain rule has to be applied

$$
\begin{aligned}
\frac{\mathrm{D} \varphi}{\mathrm{D} t} & =\frac{\partial \varphi}{\partial t}+\frac{\partial \varphi}{\partial x^{i}} \frac{\mathrm{D} x^{i}}{\mathrm{D} t} \\
& =\frac{\partial \varphi}{\partial t}+\boldsymbol{v} \cdot \boldsymbol{\nabla} \varphi .
\end{aligned}
$$

The material derivative is the local rate of change of a given particle at a given position and at a given time plus the convective rate of change related to the moving volume.

\footnotetext{
${ }^{2}$ Another common notation for the material derivative is a dot on the variable $\dot{x}$.
} 
Reynolds transport theorem for a material volume For the derivation of the balance equations we need the Reynolds transport theorem for a material volume. A mass conserving volume is called material volume (or material body) and here denoted by $V_{0}$. It is moving with time and deforming in general. A quantity $\mathcal{B}_{0}$ continuously defined over a material volume $V_{0}$ is given by $\mathcal{B}_{0}=\int_{V_{0}} \varphi \mathrm{d} V$. The rate of change of $\mathcal{B}_{0}$ with respect to time is given by

$$
\frac{\mathrm{d} \mathcal{B}_{0}(t)}{\mathrm{d} t}=\frac{\mathrm{d}}{\mathrm{d} t} \int_{V_{0}(\boldsymbol{x}, t)} \varphi(\boldsymbol{x}, t) \mathrm{d} V .
$$

In an Eulerian representation $V_{0}(\boldsymbol{x}, t)$ depends on time, so that integration and differentiation can not be interchanged. With the Jacobian $J=\operatorname{det}\left(\frac{\partial x^{i}}{\partial \xi_{j}}\right)$ the volume element can be transformed from spatial coordinates into material coordinates $\mathrm{d} V=J \mathrm{~d} V_{0}$. The material volume element $\mathrm{d} V_{0}$ does not depend on time, so that then integration and differentiation can be interchanged. The time derivative becomes the material derivative

$$
\frac{\mathrm{d}}{\mathrm{d} t} \int_{V_{0}(\boldsymbol{x}, t)} \varphi(\boldsymbol{x}, t) \mathrm{d} V=\int_{V_{0}(\boldsymbol{\xi}, t)} \frac{\mathrm{D}}{\mathrm{D} t}(\varphi(\boldsymbol{\xi}) J) \mathrm{d} V_{0} .
$$

Using the material derivative of the Jacobian $\frac{\mathrm{D} J}{\mathrm{D} t}=J \boldsymbol{\nabla} \cdot \boldsymbol{v}$ we get

$$
\int \frac{\mathrm{D}}{\mathrm{D} t}(\varphi J) \mathrm{d} V_{0}=\int\left(\frac{\mathrm{D} \varphi}{\mathrm{D} t} J+\varphi \frac{\mathrm{D} J}{\mathrm{D} t}\right) \mathrm{d} V_{0}=\int\left(\frac{\mathrm{D} \varphi}{\mathrm{D} t}+\varphi \boldsymbol{\nabla} \cdot \boldsymbol{v}\right) J \mathrm{~d} V_{0},
$$

where we have dropped the integration limits for simplicity. After transforming the volume element back into spatial variables and by using the material derivative (23) of a field variable we get for the rate of change with time of $\mathcal{B}_{0}$

$$
\begin{aligned}
\frac{\mathrm{d}}{\mathrm{d} t} \int_{V_{0}} \varphi \mathrm{d} V & =\int\left(\frac{\mathrm{D} \varphi}{\mathrm{D} t}+\varphi \boldsymbol{\nabla} \cdot \boldsymbol{v}\right) \mathrm{d} V, \\
& =\int\left(\frac{\partial \varphi}{\partial t}+\nabla \cdot[\varphi \boldsymbol{v}]\right) \mathrm{d} V .
\end{aligned}
$$

Note that when the derivative of the integral is taken the integration domain has to be indicated. By using Gauss theorem 3 , the divergence term in the volume integral can be changed into an area integral

$$
\frac{\mathrm{d}}{\mathrm{d} t} \int_{V_{0}} \varphi \mathrm{d} V=\int \frac{\partial \varphi}{\partial t} \mathrm{~d} V+\oint \varphi \boldsymbol{v} \cdot \boldsymbol{n} \mathrm{d} A
$$

\footnotetext{
${ }^{3}$ The Gauss theorem or divergence theorem for a vector $\boldsymbol{f}$ is given as: $\int \boldsymbol{\nabla} \cdot \boldsymbol{f} \mathrm{d} V=\oint \boldsymbol{f} \cdot \boldsymbol{n} \mathrm{d} A$.
} 
where the normal vector is directed outwards on the surface. The velocity $\boldsymbol{v}$ is the velocity of mass while moving across the surface. Equations (24) - (25) are called Reynolds transport theorem. In the form of (25) the Reynolds transport theorem has a physical meaning: The rate of accumulation of a quantity in a material volume can be interpreted as the rate of accumulation of the quantity in a volume that equals the material volume at a given time plus convective flux (connected to mass) leaving the volume through the surface at that time.

\subsection{Reynolds transport theorem for a material volume with an interface}

We consider a material volume $V_{0}=V_{l}+$ $V_{g}$, where the field variable has the value $\varphi_{l}$ ( $l$ for liquid phase) in the volume $V_{l}$ and the value $\varphi_{g}$ ( $g$ for gas phase) in the volume $V_{g}$, as shown in figure 3. An interface between two immiscible fluids is called a material interface, it is formed by the same material elements or particles at all times. If phase change occurs at an interface between two aggregate states of a fluid, as is the case of

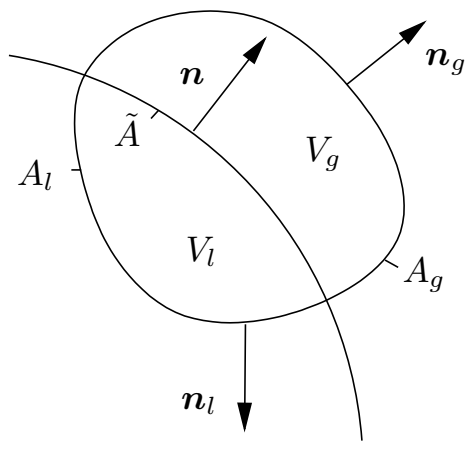
condensation or evaporation, the surface ve-

Figure 3: Material volume with interface

locity $\boldsymbol{u}$ of the interface differs from the velocity $\boldsymbol{v}$ of the mass, and the interface is called a singular interface.

The difference between the two values at the surface is denoted by

$$
\llbracket \varphi \rrbracket:=\varphi_{g}-\varphi_{l}
$$

and called the jump of $\varphi$ across the interface.

The rate of change of $\mathcal{B}_{0}$ with respect to time is the sum of the rate of change of $\mathcal{B}_{l}$ and $\mathcal{B}_{g}$ with respect to time

$$
\frac{\mathrm{d} \mathcal{B}_{0}(t)}{\mathrm{d} t}=\frac{\mathrm{d} \mathcal{B}_{l}(t)}{\mathrm{d} t}+\frac{\mathrm{d} \mathcal{B}_{g}(t)}{\mathrm{d} t}
$$

that is

$$
\frac{\mathrm{d}}{\mathrm{d} t} \int_{V_{0}} \varphi \mathrm{d} V=\frac{\mathrm{d}}{\mathrm{d} t} \int_{V_{l}} \varphi_{l} \mathrm{~d} V+\frac{\mathrm{d}}{\mathrm{d} t} \int_{V_{g}} \varphi_{g} \mathrm{~d} V .
$$

The volumes $V_{l}$ and $V_{g}$ are not material, so that we need the Reynolds transport theorem in a modified version for an arbitrary volume. 
Reynolds transport theorem for two arbitrary volumes A quantity $\mathcal{B}_{u}$ which is continuously defined over an arbitrary volume $V_{u}$ is given by $\mathcal{B}_{u}=\int_{V_{u}} \varphi \mathrm{d} V$. The volume $V_{u}$ is assumed to consist of fictive mass and shall be material (conserving the fictive mass). Then the rate of change with time of $\mathcal{B}_{u}$, according to the Reynolds transport theorem (25), is given as

$$
\frac{\mathrm{d}}{\mathrm{d} t} \int_{V_{u}} \varphi \mathrm{d} V=\int \frac{\partial \varphi}{\partial t} \mathrm{~d} V+\oint \varphi \boldsymbol{u} \cdot \boldsymbol{n} \mathrm{d} A
$$

where $\boldsymbol{u}$ is the velocity of the boundary of the considered volume.

Applying the general formula to the two control volumes together with (26) yields Reynolds transport theorems for each of the two volumes $V_{l}$ and $V_{g}$ as

$$
\frac{\mathrm{d}}{\mathrm{d} t} \int_{V_{l}} \varphi \mathrm{d} V=\int_{V_{l}} \frac{\partial \varphi}{\partial t} \mathrm{~d} V+\int_{A_{l}} \varphi \boldsymbol{v} \cdot \mathrm{d} \boldsymbol{A}+\int_{\tilde{A}} \varphi_{l} \boldsymbol{u} \cdot \boldsymbol{n} \mathrm{d} \tilde{A},
$$

and

$$
\frac{\mathrm{d}}{\mathrm{d} t} \int_{V_{g}} \varphi \mathrm{d} V=\int_{V_{g}} \frac{\partial \varphi}{\partial t} \mathrm{~d} V+\int_{A_{g}} \varphi \boldsymbol{v} \cdot \mathrm{d} \boldsymbol{A}+\int_{\tilde{A}} \varphi_{g} \boldsymbol{u} \cdot(-\boldsymbol{n}) \mathrm{d} \tilde{A} .
$$

By adding (27) and (28) we obtain a Reynolds transport theorem for the entire volume $V_{0}=V_{l}+V_{g}$ as

$$
\frac{\mathrm{d}}{\mathrm{d} t} \int_{V_{0}} \varphi \mathrm{d} V=\int \frac{\partial \varphi}{\partial t} \mathrm{~d} V+\oint \varphi \boldsymbol{v} \cdot \mathrm{d} \boldsymbol{A}-\int_{\tilde{A}} \llbracket \varphi \rrbracket \boldsymbol{u} \cdot \boldsymbol{n} \mathrm{d} \tilde{A} .
$$

Equation (29) is the Reynolds transport theorem for a material volume with a singular interface. It states that the rate of accumulation of a quantity in a material volume, where $\varphi$ undergoes a jump on an interface can be interpreted as the rate of accumulation of the quantity in a volume that equals the material volume at a given time plus convective flow of the quantities $\varphi_{l}$ and $\varphi_{g}$ leaving the volume through the outer surface and the interface at that time. Here again the integration limits are dropped where the integrals are evaluated at a given time. Only the integration domain of the integral along the interface $\tilde{A}$ has to be indicated. 
By using Gauss theorem 4 , the Reynolds transport theorem (29) for a material volume with a singular interface can be rewritten as

$$
\frac{\mathrm{d}}{\mathrm{d} t} \int_{V_{0}} \varphi \mathrm{d} V=\int\left(\frac{\partial \varphi}{\partial t}+\nabla \cdot[\varphi \boldsymbol{v}]\right) \mathrm{d} V+\int_{\tilde{A}} \llbracket \varphi(\boldsymbol{v}-\boldsymbol{u}) \rrbracket \cdot \boldsymbol{n} \mathrm{d} \tilde{A} .
$$

\subsection{Kinematics of a moving interface and velocities}

In this section we present the kinematics of the moving interface and discuss the material (or convected) surface derivative, fluid velocity and interface velocity.

Kinematics of the moving interface If we consider phase change, the interface is not composed of a fixed set of particles, there will be mass transfer between the interface and the two adjoining phases. According to the basic assumption of continuum theory a surface consists at every moment of infinitely many particles. In particular, a particle joining the interface coincides with the particle that was at that position before. For a particle which is leaving the interface instantaneously another particle emerges. So although there is a many-to-one mapping between particles and the region in the surface that is occupied by them, we can assign one representing particle for all possible particles at one point. We will call the representing particle simply particle (see [TrTo]).

The position vector to a point on the surface was given in section 2 as a function of surface coordinates and time (11) and is here denoted by a lower index $S$

$$
\boldsymbol{x}_{S}=\boldsymbol{x}_{S}\left(u^{\alpha}, t\right) \quad \text { with } \alpha=1,2 .
$$

At a given time a particle on the surface is represented by a position vector, which is here also denoted by a lower index $S$

$$
\boldsymbol{\xi}_{S}=\boldsymbol{\xi}_{S}\left(u_{0}^{\alpha}\right) .
$$

We take this position as the initial position and call it intrinsic surface reference configuration. Conversely at a given time every position in the surface corresponds to a particle

$$
u_{0}^{\alpha}=u_{0}^{\alpha}\left(\boldsymbol{\xi}_{S}\right)
$$

\footnotetext{
${ }^{4}$ For a material volume with an internal interface Gauss theorem becomes (compare footnote 3) $\int \boldsymbol{\nabla} \cdot[\varphi \boldsymbol{v}] \mathrm{d} V=\oint \varphi \boldsymbol{v} \cdot \mathrm{d} \boldsymbol{A}-\int_{\tilde{A}} \llbracket \varphi \rrbracket \boldsymbol{v} \cdot \boldsymbol{n} \mathrm{d} \tilde{A}$.
} 
Obviously the initial position of a particle in the surface can be identified either by (32) or by (33). At another time the particle is given by another set of coordinates as a function of the reference configuration of the particle and time

$$
u^{\alpha}=u^{\alpha}\left(u_{0}^{\alpha}, t\right) .
$$

With the assumption of a representing surface particle we established a one-to-one mapping between the coordinates of a surface particle and the surface coordinates, so that we can write reversely

$$
u_{0}^{\alpha}=u_{0}^{\alpha}\left(u^{\alpha}, t\right) .
$$

Equations (34) and (35) describe the intrinsic motion of a surface particle within the surface, without knowing how the surface itself is moving.

The motion of a surface particle in space we get from the motion of the surface (31) and the intrinsic motion of the surface particles on the surface (34), (35) as

$$
\boldsymbol{x}_{S}=\boldsymbol{x}_{S}\left(u_{0}^{\alpha}, t\right) \quad \text { or } \quad \boldsymbol{x}_{S}=\boldsymbol{x}_{S}\left(u^{\alpha}\left(u_{0}^{\alpha}, t\right), t\right) .
$$

Equation (36) is not reversible in general. A position in space is corresponding to every surface particle, but the converse is not true (as explained for phase change).

A surface field variable is here denoted by $\varphi_{S}$. It can be given with (31) as a function of space and time

$$
\varphi_{S}=\varphi_{S}\left(\boldsymbol{x}_{S}, t\right) . \quad \text { or } \quad \varphi_{S}=\varphi_{S}\left(u^{\alpha}, t\right) .
$$

Or it can be given with (236) as a function of particle and time

$$
\varphi_{S}=\varphi_{S}\left(u_{0}^{\alpha}, t\right) \quad \text { or } \quad \varphi_{S}=\varphi_{S}\left(u^{\alpha}\left(u_{0}^{\alpha}, t\right), t\right) \text {. }
$$

Material surface derivative The partial derivative of a surface field variable with respect to time where the material surface coordinates are held constant is called material (or convected) surface derivative and it is here denoted with $\mathrm{D}_{S}$

$$
\frac{\mathrm{D}_{S} \varphi_{S}}{\mathrm{D} t}=\frac{\partial \varphi_{S}\left(u_{0}^{\alpha}, t\right)}{\partial t}=\left(\frac{\partial \varphi_{S}}{\partial t}\right)_{u_{0}^{\alpha}} .
$$

It is the rate of change of a surface field variable with respect to time an observer moving with a surface particle would see. The material surface derivative of a position vector is the velocity of a given surface particle

$$
\boldsymbol{v}=\frac{\mathrm{D}_{S} \boldsymbol{x}_{S}}{\mathrm{D} t} .
$$


Here we did not denote $\boldsymbol{v}$ with a lower index $S$ to be consistent with the balance equations as they will be given later. Surface balance equations are often conveniently given in spatial surface coordinates. To obtain the surface material derivative of a surface field variable $\varphi_{S}\left(u^{\alpha}\left(u_{0}^{\alpha}, t\right), t\right)$ in spatial surface coordinates the chain rule has to be applied

$$
\frac{\mathrm{D}_{S} \varphi_{S}}{\mathrm{D} t}=\frac{\partial \varphi_{S}}{\partial t}+\frac{\partial \varphi_{S}}{\partial u^{\alpha}} \frac{\mathrm{D} u^{\alpha}}{\mathrm{D} t}
$$

With the surface gradient (11)

$$
\nabla_{S}=\boldsymbol{a}^{\alpha} \frac{\partial}{\partial u^{\alpha}}
$$

and the intrinsic surface velocity

$$
\boldsymbol{w}=\frac{\mathrm{D} u^{\alpha}}{\mathrm{D} t} \boldsymbol{a}_{\alpha}
$$

the material surface derivative becomes

$$
\frac{\mathrm{D}_{S} \varphi_{S}}{\mathrm{D} t}=\frac{\partial \varphi_{S}}{\partial t}+\boldsymbol{w} \cdot \nabla_{S} \varphi_{S}
$$

The material surface derivative is the local rate of change at a position of a given surface particle at a given time plus the convective rate of change related to the moving surface.

Velocity of an interface particle relative to the velocity of a moving interface The material surface derivative of the surface position vector $\boldsymbol{x}_{S}\left(u^{\alpha}\left(u_{0}^{\alpha}, t\right), t\right)$ is by (39) given as

$$
\frac{\mathrm{D}_{S} \boldsymbol{x}_{S}}{\mathrm{D} t}=\frac{\partial \boldsymbol{x}_{S}}{\partial t}+\boldsymbol{w} \cdot \boldsymbol{\nabla}_{S} \boldsymbol{x}_{S} .
$$

The partial derivative of the surface position vector with respect to time (where $u^{\alpha}$ held constant) is the velocity of the moving interface

$$
\boldsymbol{u}=\frac{\partial \boldsymbol{x}_{S}}{\partial t} .
$$

For the second term on the right hand side we have $(\boldsymbol{w} \cdot \boldsymbol{\nabla}) \boldsymbol{x}_{S}=\boldsymbol{w}\left(\boldsymbol{\nabla}_{S} \otimes \boldsymbol{x}_{S}\right)$ and the surface gradient (11) of the surface position vector is the surface identity tensor

$$
\boldsymbol{\nabla}_{S} \otimes \boldsymbol{x}_{S}=\boldsymbol{a}^{\alpha} \frac{\partial}{\partial u^{\alpha}} \otimes \boldsymbol{x}_{S}=\boldsymbol{a}^{\alpha} \otimes \frac{\partial \boldsymbol{x}_{S}}{\partial u^{\alpha}}=\boldsymbol{a}^{\alpha} \otimes \boldsymbol{a}_{\alpha}=\boldsymbol{I}_{S},
$$

so that the material derivative of the surface position vector becomes

$$
\begin{aligned}
\frac{\mathrm{D}_{S} \boldsymbol{x}_{S}}{\mathrm{D} t} & =\frac{\partial \boldsymbol{x}_{S}}{\partial t}+\boldsymbol{w} \cdot \nabla_{S} \boldsymbol{x}_{S}, \\
\boldsymbol{v} & =\boldsymbol{u}+\boldsymbol{w} .
\end{aligned}
$$


The intrinsic surface velocity is the velocity of a surface particle relative to the velocity of the surface

$$
\boldsymbol{w}=\boldsymbol{v}-\boldsymbol{u} .
$$

Note that in general $\boldsymbol{u}$ has a normal and a tangential part, so that $\boldsymbol{w}$ is not necessarily the tangential part of $\boldsymbol{v}$.

Surface velocity for an implicit surface parametrization In section 2.3 we discussed the geometrical properties of a surface defined by an implicit function $F\left(\boldsymbol{x}_{S}\left(u^{\alpha}, t\right), t\right)=0$. Differentiating $F=0$ with respect to time gives

$$
\frac{\partial F}{\partial t}+\frac{\partial F}{\partial x^{i}} \frac{\partial x^{i}}{\partial t}=0 \quad \text { and equivalently } \quad \frac{\partial F}{\partial t}+\boldsymbol{u} \cdot \nabla F=0,
$$

where we dropped the subscript $S$ at $\boldsymbol{x}_{S}$ for simplicity.

With the normal vector $\boldsymbol{n}=\frac{\boldsymbol{\nabla} F}{|\boldsymbol{\nabla} F|}$ as derived in (15) we can write either

$$
\boldsymbol{u} \cdot \boldsymbol{n}=-\frac{\frac{\partial F}{\partial t}}{|\boldsymbol{\nabla} F|} \quad \text { or } \quad \boldsymbol{u} \cdot \boldsymbol{n}=\boldsymbol{u} \cdot \frac{\boldsymbol{\nabla} F}{|\boldsymbol{\nabla} F|} .
$$

The first equation is independent of the parametrization, so that all possible surface velocities have the same normal component $\boldsymbol{u} \cdot \boldsymbol{n}$, which is called the speed of displacement. It is convenient to choose a parametrization such that the surface velocity becomes the surface normal velocity

$$
\boldsymbol{u}=(\boldsymbol{n} \otimes \boldsymbol{n}) \boldsymbol{u}=(\boldsymbol{u} \cdot \boldsymbol{n}) \boldsymbol{n} .
$$

The surface defined by $F(u, v, z, t)=z-h(u, v, t)$ has the surface position vector

$$
\boldsymbol{x}_{S}(u, v, t)=u \boldsymbol{e}_{x}+v \boldsymbol{e}_{y}+h(u, v, t) \boldsymbol{e}_{z} .
$$

For an implicit surface parametrization the surface velocity is the surface normal velocity and is given by

$$
\boldsymbol{u}=\frac{\partial \boldsymbol{x}_{S}}{\partial t}=\left[\begin{array}{c}
0 \\
0 \\
\frac{\partial h}{\partial t}
\end{array}\right] .
$$

Dotting (43) with $\boldsymbol{n}$ gives

$$
\begin{aligned}
\boldsymbol{v}_{s} \cdot \boldsymbol{n} & =\boldsymbol{u} \cdot \boldsymbol{n}+\frac{\mathrm{D} u^{\alpha}}{\mathrm{D} t} \boldsymbol{a}_{\alpha} \cdot \boldsymbol{n} \\
& =\boldsymbol{u} \cdot \boldsymbol{n} .
\end{aligned}
$$




\subsection{Reynolds transport theorem and divergence theorem for a surface}

In the balance equations we also need the Reynolds transport theorem for an interface, which is not material if we allow phase change. However, we can always assume the interface to be composed of a fixed set of particles, as in section 3.3. For a quantity $\mathcal{S}_{0}$ continuously defined over such an interface $\tilde{A}_{0}$, we write $\mathcal{S}_{0}=\int_{\tilde{A}_{0}} \varphi_{S} \mathrm{~d} \tilde{A}$. The rate of change of $\mathcal{S}_{0}$ with respect to time is given by

$$
\frac{\mathrm{d} \mathcal{S}_{0}(t)}{\mathrm{d} t}=\frac{\mathrm{d}}{\mathrm{d} t} \int_{\tilde{A}_{0}\left(\boldsymbol{x}_{S}, t\right)} \varphi_{S}\left(\boldsymbol{x}_{S}, t\right) \mathrm{d} \tilde{A} .
$$

In an Eulerian representation $\tilde{A}_{0}\left(\boldsymbol{x}_{S}, t\right)$ depends on time. As for the Reynolds transport theorem for a material volume the area element is transformed with the surface Jacobian determinant $j=\operatorname{det}\left(\frac{\partial x_{S}^{i}}{\partial \xi_{S}^{i}}\right)$ from spatial coordinates into material coordinates $\mathrm{d} \tilde{A}=j \mathrm{~d} \tilde{A}_{0}$. Then $\tilde{A}_{0}\left(\boldsymbol{\xi}_{S}\right)$ does not depend on time and integration and differentiation can be interchanged, so that the time derivative becomes the material surface derivative (39)

$$
\frac{\mathrm{d}}{\mathrm{d} t} \int_{A_{0}} \varphi_{S} \mathrm{~d} \tilde{A}=\int \frac{\mathrm{D}_{S}}{\mathrm{D} t}\left(\varphi_{S} j\right) \mathrm{d} \tilde{A}_{0} .
$$

Using the material surface derivative of the surface Jacobian $\frac{\mathrm{D}_{S} j}{\mathrm{D} t}=j \boldsymbol{\nabla}_{S} \cdot \boldsymbol{v}_{S}$ we get

$$
\int \frac{\mathrm{D}_{S}}{\mathrm{D} t}\left(\varphi_{S} j\right) \mathrm{d} \tilde{A}_{0}=\int\left(\frac{\mathrm{D}_{S} \varphi_{S}}{\mathrm{D} t} j+\varphi_{S} \frac{\mathrm{D}_{S} j}{\mathrm{D} t}\right) \mathrm{d} \tilde{A}_{0}=\int\left(\frac{\mathrm{D}_{S} \varphi_{S}}{\mathrm{D} t}+\varphi_{S} \boldsymbol{\nabla} \cdot \boldsymbol{v}_{S}\right) j \mathrm{~d} \tilde{A}_{0} .
$$

After transforming the area element back into spatial coordinates and by using the material surface derivative (39) with the relative velocity (44) we get for the rate of change of $\mathcal{S}_{0}$ with respect to time

$$
\begin{aligned}
\frac{\mathrm{d}}{\mathrm{d} t} \int_{\tilde{A}_{0}} \varphi_{S} \mathrm{~d} \tilde{A} & =\int\left(\frac{\mathrm{D}_{S} \varphi_{S}}{\mathrm{D} t}+\varphi_{S} \boldsymbol{\nabla} \cdot \boldsymbol{v}_{S}\right) \mathrm{d} \tilde{A} \\
& =\int\left(\frac{\partial \varphi_{S}}{\partial t}+\left[\boldsymbol{v}_{S}-\boldsymbol{u}\right] \cdot \boldsymbol{\nabla}_{S} \varphi_{S}+\varphi_{S} \boldsymbol{\nabla} \cdot \boldsymbol{v}_{S}\right) \mathrm{d} \tilde{A} \\
& =\int\left(\frac{\partial \varphi_{S}}{\partial t}+\nabla_{S} \cdot\left[\varphi_{S} \boldsymbol{v}_{S}\right]-\boldsymbol{u} \cdot \boldsymbol{\nabla}_{S} \varphi_{S}\right) \mathrm{d} \tilde{A}
\end{aligned}
$$

This is the Reynolds transport theorem for surfaces. Comparing the last equation with the Reynolds transport theorem for material volumes (24) shows an additional term related to the moving surface. 
The last equation of (48) can be transformed further. In this section we derive the divergence theorem for surfaces, which will be used here and again in section 4.3, so that we derive it using the abbreviation $\boldsymbol{f}=\varphi_{S} \boldsymbol{v}_{S}$. Splitting $\boldsymbol{f}$ in normal and tangential part the area integral of the surface divergence of $\boldsymbol{f}$ becomes

$$
\int \boldsymbol{\nabla}_{S} \cdot \boldsymbol{f} \mathrm{d} \tilde{A}=\int \boldsymbol{\nabla}_{S} \cdot[(\boldsymbol{f} \cdot \boldsymbol{n}) \boldsymbol{n}] \mathrm{d} \tilde{A}+\int \boldsymbol{\nabla}_{S} \cdot[(\boldsymbol{f} \cdot \boldsymbol{b}) \boldsymbol{b}] \mathrm{d} \tilde{A} .
$$

Applying Product Rule to the first integrand on the right hand side gives

$$
\boldsymbol{\nabla}_{S} \cdot[(\boldsymbol{f} \cdot \boldsymbol{n}) \boldsymbol{n}]=\underbrace{\boldsymbol{n} \cdot \boldsymbol{\nabla}_{S}}_{=0}(\boldsymbol{f} \cdot \boldsymbol{n})+(\boldsymbol{f} \cdot \boldsymbol{n}) \boldsymbol{\nabla}_{S} \cdot \boldsymbol{n}=-2 H \boldsymbol{f} \cdot \boldsymbol{n},
$$

where we invoked the definition of mean curvature (13) in section 2.2 , and the fact that the surface gradient (9) is perpendicular to the normal vector on the surface.

The second integral is an intrinsic area integral. The binormal vector $\boldsymbol{b}=\boldsymbol{t} \times \boldsymbol{n}$ is perpendicular to both the tangential vector $\boldsymbol{t}$ along the curve and to the surface normal vector, and therefore is the outward normal to the boundary curve as shown in figure 4 . Using Stokes theorem 5 to transform the area integral into a line integral

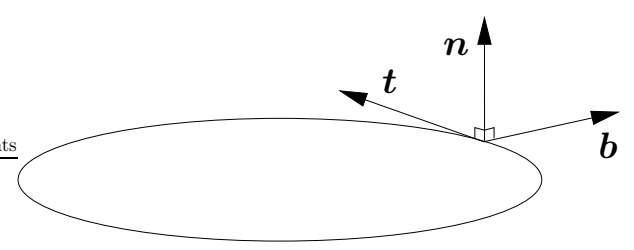

Figure 4: Base vectors on a bounded surface

$$
\int \boldsymbol{\nabla}_{S} \cdot[(\boldsymbol{f} \cdot \boldsymbol{b}) \boldsymbol{b}] \mathrm{d} \tilde{A}=\oint(\boldsymbol{f} \cdot \boldsymbol{b}) \mathrm{d} \tilde{C} .
$$

equation (49) becomes

$$
\int \boldsymbol{\nabla}_{S} \cdot \boldsymbol{f} \mathrm{d} \tilde{A}=-\int 2 H \boldsymbol{f} \cdot \boldsymbol{n} \mathrm{d} \tilde{A}+\oint \boldsymbol{f} \cdot \boldsymbol{b} \mathrm{d} \tilde{C} .
$$

This equation is called the divergence theorem for surfaces. (It should not be confused with the divergence theorem in footnote 5.)

Alternative version of the Reynolds transport theorem for surfaces Substituting (50) into the last equation of (48) gives an alternative version of the Reynolds transport theorem for surfaces

$$
\frac{\mathrm{d}}{\mathrm{d} t} \int_{\tilde{A}_{0}} \varphi_{S} \mathrm{~d} \tilde{A}=\int\left(\frac{\partial \varphi_{S}}{\partial t}-\boldsymbol{u} \cdot \nabla_{S} \varphi_{S}-2 H \varphi_{S} \boldsymbol{v}_{S} \cdot \boldsymbol{n}\right) \mathrm{d} \tilde{A}+\oint \varphi_{S} \boldsymbol{v}_{S} \cdot \boldsymbol{b} \mathrm{d} \tilde{C} .
$$

\footnotetext{
${ }^{5}$ Stokes theorem entirely defined in surface vectors is given as: $\int \boldsymbol{\nabla}_{S} \cdot \boldsymbol{f} \mathrm{d} A=\oint \boldsymbol{f} \cdot \boldsymbol{b} \mathrm{d} C$.
} 
The rate of accumulation of a quantity in a surface can be interpreted as the rate of accumulation of the quantity in a material surface that equals the surface at a given time plus flux arising from the moving surface, plus convective flux normal to the surface (curvature term) and convective flux through the boundary curve of the area.

\section{Generic model equations for two-phase flows with surface tension}

In this section we derive the generic model equations for a problem with a moving interface. First we recall the balance equations for incompressible fluids and then we derive a differential balance equation for a moving interface between two fluids, a so called jump condition. This interface balance equation includes phase change, but to also include surface tension, a balance equation for the interface itself has to be formulated, which is then added to the balance equation for the bulk phases.

The material about balance equations in general is mainly based on BiStLi] and S199. Further on $[\mathrm{De}]$ and $[\mathrm{Hu}]$. The material about interface balance equations is mainly based on [TrTo], [Sl07] and [Ed]. Some recent references on two phase flow problems are [IsHi], [Ko] and $\mathrm{GaPr}$.

\subsection{Balance equations for bulk fluids}

Balance equations are formulated for physical quantities that are continuously defined over a spatial region (for instances a volume), such as mass, momentum or energy. We denote those quantities by $\mathcal{B}=\int_{V} \varphi \mathrm{d} V$.

Balance equation for a material volume A material (mass conserving) volume $V_{0}$ is in general moving with time. A balance equation for a physical quantity $\mathcal{B}_{0}=\int_{V_{0}} \varphi \mathrm{d} V$ in a material volume states that the rate of accumulation of the quantity in the material volume is given by an conductive flux of the quantity (not connected to mass) that enters the volume across the surface plus supply of the quantity to the material volume 6

$$
\frac{\mathrm{d}}{\mathrm{d} t} \int_{V_{0}} \varphi \mathrm{d} V=-\oint \boldsymbol{\zeta} \cdot \mathrm{d} \boldsymbol{A}+\int \pi \mathrm{d} V,
$$

\footnotetext{
${ }^{6}$ Some authors distinguish between supply of quantity to the volume and production of quantity within the volume. Then conservation equations can be defined as balance equations without a production term. However, it is more intuitive to distinguish only between surface terms and volume terms.
} 
where $\boldsymbol{\zeta}$ is the efflux density and $\pi$ is the supply density. The surface element vector $\mathrm{d} \boldsymbol{A}=$ $\boldsymbol{n} \mathrm{d} A$ is directed outwards, normal to the surface. As before we drop the integration limits except in the case, where the derivative of the integral is taken. With the Reynolds transport theorem (24) the balance equation (52) for a material volume becomes

$$
\int\left(\frac{\partial \varphi}{\partial t}+\nabla \cdot[\varphi \boldsymbol{v}]\right) \mathrm{d} V=-\oint \boldsymbol{\zeta} \cdot \mathrm{d} \boldsymbol{A}+\int \pi \mathrm{d} V
$$

Balance equation for a stationary volume To derive a balance equation for a stationary volume the Reynolds transport theorem in the form of (25) is substituted for the right hand side of (52)

$$
\int \frac{\partial \varphi}{\partial t} \mathrm{~d} V+\oint \varphi \boldsymbol{v} \cdot \mathrm{d} \boldsymbol{A}=-\oint \boldsymbol{\zeta} \cdot \mathrm{d} \boldsymbol{A}+\int \pi \mathrm{d} V .
$$

Now the integration domain of the volume integral on the left hand side is constant and differentiation and integration can be interchanged. This gives the balance equation for a stationary volume

$$
\frac{\mathrm{d}}{\mathrm{d} t} \int \varphi \mathrm{d} V=-\oint \varphi \boldsymbol{v} \cdot \mathrm{d} \boldsymbol{A}-\oint \boldsymbol{\zeta} \cdot \mathrm{d} \boldsymbol{A}+\int \pi \mathrm{d} V .
$$

This equation has again a physical meaning: The accumulation of $\mathcal{B}=\int \varphi \mathrm{d} V$ in a stationary volume is given by convective and conductive flux of quantity across the surface to the volume plus supply of quantity to the volume.

Differential balance equation For numerical computations a differential version of the balance equation is preferable. The starting point is equation (53)

$$
\int\left(\frac{\partial \varphi}{\partial t}+\nabla \cdot[\varphi \boldsymbol{v}]\right) \mathrm{d} V=-\oint \boldsymbol{\zeta} \cdot \mathrm{d} \boldsymbol{A}+\int \pi \mathrm{d} V
$$

Using the divergence theorem (see footnote 3 in section 3.1) for the surface integral, we get

$$
\int\left(\frac{\partial \varphi}{\partial t}+\nabla \cdot[\varphi \boldsymbol{v}+\boldsymbol{\zeta}]-\pi\right) \mathrm{d} V=0
$$

This equation must hold for any arbitrary volume so that we get the differential balance equation

$$
\frac{\partial \varphi}{\partial t}+\nabla \cdot(\varphi \boldsymbol{v})=-\nabla \cdot \zeta+\pi
$$

Although this equation represent the same physical phenomenon as before (accumulation, flux, supply) the various terms cannot be interpreted in the same way as the integral balance equations. 


\subsection{Jump conditions at an interface between two fluids}

Next we derive balance equations for an interface between two homogeneous bulk phases with phase change (condensation or evaporation).

Balance equation for a material volume with a singular interface The balance equation (52) for a quantity $\mathcal{B}_{0}$ in a material volume

$$
\frac{\mathrm{d}}{\mathrm{d} t} \int_{V_{0}} \varphi \mathrm{d} V=-\oint \boldsymbol{\zeta} \cdot \mathrm{d} \boldsymbol{A}+\int \pi \mathrm{d} V
$$

holds for a material volume whether or not the volume has a singular interface. The rate of accumulation in $V_{0}=V_{l}+V_{g}$ is the sum of the rate of accumulation in the volumes $V_{l}$ and $V_{g}$

$$
\frac{\mathrm{d}}{\mathrm{d} t} \int_{V_{0}} \varphi \mathrm{d} V=\frac{\mathrm{d}}{\mathrm{d} t} \int_{V_{l}} \varphi \mathrm{d} V+\frac{\mathrm{d}}{\mathrm{d} t} \int_{V_{g}} \varphi \mathrm{d} V
$$

The volumes $V_{l}$ and $V_{g}$ are not material, so we use Reynolds transport theorem for an arbitrary volume with a singular interface (30). Substituting (30) for the left hand side of (52) gives the balance equation for a material volume with a singular interface

$$
\int\left(\frac{\partial \varphi}{\partial t}+\nabla \cdot[\varphi \boldsymbol{v}]\right) \mathrm{d} V+\int_{\tilde{A}} \llbracket \varphi(\boldsymbol{v}-\boldsymbol{u}) \rrbracket \cdot \boldsymbol{n} \mathrm{d} \tilde{A}=-\oint \boldsymbol{\zeta} \cdot \mathrm{d} \boldsymbol{A}+\int \pi \mathrm{d} V .
$$

The double brackets denote again the difference between the two values at the surface.

To derive a differential form of (56), a special volume in a form of a small box is considered, which is moving together with the interface as shown in figure 5. Two faces of the box are parallel to the interface. By taking the limit of the shorter side faces $A_{h} \rightarrow 0$ the volume integrals vanish and $A_{l}$ and $A_{g}$ merge with $\tilde{A}$. For the volume integrals to vanish their integrands must be limited (but not necessary continuous). Then the normal vectors of the two outer faces of the box

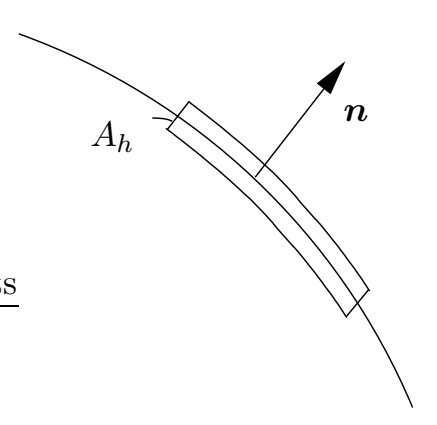

Figure 5: Volume in form of a box 
transform into either $\boldsymbol{n}$ or $\boldsymbol{- n}$ and only the surface integral over the interface $\tilde{A}$ remains

$$
\int_{\tilde{A}}\left(\llbracket \varphi(\boldsymbol{v}-\boldsymbol{u}) \rrbracket \cdot \boldsymbol{n}+\left[\boldsymbol{\zeta}_{l} \cdot(-\boldsymbol{n})-\boldsymbol{\zeta}_{g} \cdot \boldsymbol{n}\right]\right) \mathrm{d} \tilde{A}=0 .
$$

The integral must hold for any arbitrary surface so that the integrand must be zero

$$
\llbracket \varphi[\boldsymbol{v}-\boldsymbol{u}] \cdot \boldsymbol{n} \rrbracket+\llbracket \boldsymbol{\zeta} \cdot \boldsymbol{n} \rrbracket=0 .
$$

Equation (57) is called a jump condition and describes the phase change at the interface and the conductive flux across the interface [S107.

This jump condition describes phase change and conductive flux across the surface, but it does not allow for modeling intrinsic surface properties such as surface tension (we took the limit of $\left.A_{h} \rightarrow 0\right)$.

\subsection{Balance equation and jump condition including surface tension}

Based on the assumption that a fluid interface is actually a three-dimensional region with a thickness of maybe one or more molecule diameters, the effect of the interface on the adjoining bulk fluids can be represented by assuming a two-dimensional interface consisting of surface excess mass. Surface mass is assumed to have similar properties as three-dimensional mass, such as surface density, surface viscosity, surface tension and so on. Then, analogous to the balance equation of three dimensional continua, a balance equation for the interface can be given. Adding this derived interface balance equation to the balance equation for a material volume with a singular interface gives a balance equation which includes surface tension.

Balance equation for a surface The interface between condensate and vapor is not material, the fluid velocity differs from the velocity of the surface. Nevertheless a balance equation similar to the balance equation (152) for a material volume can be given, as explained in section 3.3 . For a quantity $\mathcal{S}_{0}$ continuously defined over a surface $\tilde{A}$ we write $\mathcal{S}_{0}=\int_{\tilde{A}} \varphi_{S} \mathrm{~d} \tilde{A}$. Then a balance equation for $\mathcal{S}_{0}$ states that the rate of accumulation of surface quantity in the surface $\tilde{A}$ is given by conductive flux of surface quantity across the boundary curve of the 
surface plus supply of surface quantity at the surface

$$
\frac{\mathrm{d}}{\mathrm{d} t} \int_{\tilde{A}} \varphi_{S} \mathrm{~d} \tilde{A}=-\oint \boldsymbol{\zeta}_{S} \cdot \mathrm{d} \tilde{\boldsymbol{C}}+\int \pi_{S} \mathrm{~d} \tilde{A},
$$

where $\boldsymbol{\zeta}_{S}$ is the surface flux density and $\pi_{S}$ is the surface supply density. The line element vector $\mathrm{d} \tilde{\boldsymbol{C}}=\boldsymbol{m} \mathrm{d} \tilde{\boldsymbol{C}}$ is directed outwards normal on the boundary curve, see figure 4 in section 3.4, Using Reynolds theorem for a surface (48), equation (58) becomes

$$
\int\left(\frac{\partial \varphi_{S}}{\partial t}+\nabla_{S} \cdot\left[\varphi_{S} \boldsymbol{v}_{S}\right]-\boldsymbol{u} \cdot \nabla_{S} \varphi_{S}\right) \mathrm{d} \tilde{A}=-\oint \boldsymbol{\zeta}_{S} \cdot \mathrm{d} \tilde{\boldsymbol{C}}+\int \pi_{S} \mathrm{~d} \tilde{A}
$$

Differential balance equation for a surface To derive a differential version of (59) we transform the line integral into an area integral using the surface divergence theorem (50)

$$
\int\left(\frac{\partial \varphi_{S}}{\partial t}+\nabla_{S} \cdot\left[\varphi_{S} \boldsymbol{v}_{S}\right]-\boldsymbol{u} \cdot \nabla_{S} \varphi_{S}+\left[\boldsymbol{\nabla}_{S} \cdot \boldsymbol{\zeta}_{S}+2 H \boldsymbol{\zeta}_{S} \cdot \boldsymbol{n}\right]-\pi_{S}\right) \mathrm{d} \tilde{A}=0,
$$

where $H$ is the mean curvature. This equation must hold for any arbitrary area so that the differential surface balance equation is given as

$$
\frac{\partial \varphi_{S}}{\partial t}+\nabla_{S} \cdot\left[\varphi_{S} \boldsymbol{v}_{S}\right]-\boldsymbol{u} \cdot \boldsymbol{\nabla}_{S} \varphi_{S}=-\left[\boldsymbol{\nabla}_{S} \cdot \boldsymbol{\zeta}_{S}+2 H \boldsymbol{\zeta}_{S} \cdot \boldsymbol{n}\right]+\pi_{S}
$$

Balance equation including phase change and surface tension Adding the surface balance equation (59) to the balance equation for a material volume with a singular interface (56) gives a balance equation that includes phase change and surface tension

$$
\begin{aligned}
\int\left(\frac{\partial \varphi}{\partial t}+\nabla \cdot[\varphi \boldsymbol{v}]\right) \mathrm{d} V+\int_{\tilde{A}} & {[\varphi(\boldsymbol{v}-\boldsymbol{u}) \rrbracket \cdot \boldsymbol{n} \mathrm{d} \tilde{A}} \\
+\int\left(\frac{\partial \varphi_{S}}{\partial t}+\nabla_{S} \cdot\left[\varphi_{S} \boldsymbol{v}_{S}\right]-\boldsymbol{u} \cdot \boldsymbol{\nabla}_{S} \varphi_{S}\right) \mathrm{d} \tilde{A} & \\
& =-\oint \boldsymbol{\zeta} \cdot \mathrm{d} \boldsymbol{A}-\oint \boldsymbol{\zeta}_{S} \cdot \mathrm{d} \tilde{\boldsymbol{C}}+\int \pi \mathrm{d} V+\int \pi_{S} \mathrm{~d} \tilde{A} .
\end{aligned}
$$

Jump condition including phase change and surface tension From (61) we derive a jump condition in the same way as discussed in the last section. Transforming the line integral into an area integral using the surface divergence theorem (50) and considering a small volume 
enclosing the interface which we let shrink into a surface gives

$$
\begin{aligned}
\llbracket \varphi[\boldsymbol{v}-\boldsymbol{u}] \cdot \boldsymbol{n} \rrbracket+\llbracket \boldsymbol{\zeta} \cdot \boldsymbol{n} \rrbracket=-\frac{\partial \varphi_{S}}{\partial t}-\boldsymbol{\nabla}_{S} \cdot\left[\varphi_{S} \boldsymbol{v}_{S}\right]+\boldsymbol{u} \cdot \boldsymbol{\nabla}_{S} \varphi_{S} \\
-\underline{\left[\boldsymbol{\nabla}_{S} \cdot \boldsymbol{\zeta}_{S}+2 H \boldsymbol{\zeta}_{S} \cdot \boldsymbol{n}\right]}+\pi_{S} .
\end{aligned}
$$

If there is no material accumulation (no mass) in the surface, the surface density variables $\varphi_{S}$ and $\pi_{S}$ are zero and from the right hand side, only the underlined terms remain:

$$
\llbracket \varphi[\boldsymbol{v}-\boldsymbol{u}] \cdot \boldsymbol{n} \rrbracket+\llbracket \boldsymbol{\zeta} \cdot \boldsymbol{n} \rrbracket=-\boldsymbol{\nabla}_{S} \cdot \boldsymbol{\zeta}_{S}-2 H \boldsymbol{n} \cdot \boldsymbol{\zeta}_{S} .
$$

This jump condition describes conductive flux across the interface, phase change and surface tension. We made no additional assumption other than assume a continuous surface, in particular we do not allow the interface to break off.

\section{Model equations with phase change and surface tension}

In this section the balance equations for mass, momentum and energy for two incompressible fluids and for the interface between them are obtained and we discuss appropriate simplifications. In the last section we summarize the deduced system of partial differential equations including the jump conditions and discuss boundary conditions for the system of partial differential equations.

\subsection{Mass, momentum and energy equation}

Mass The mass balance equation for an incompressible fluid is given by (55) with $\varphi=\rho$ and $\zeta=\pi=0$

$$
\frac{\partial \rho}{\partial t}+\nabla \cdot(\rho \boldsymbol{v})=0
$$

which gives with the assumption of constant density

$$
\boldsymbol{\nabla} \cdot \boldsymbol{v}=0
$$

If the Mach number of a fluid is small compared to unity the fluid can be considered as an incompressible fluid [BiStLi].

For momentum and energy another balance equation which makes use of (63) is more preferable. For that we substitute $\varphi$ in (55) by $\rho \psi$, apply product rule on both terms on the 
left side and receive

$$
\frac{\partial(\rho \psi)}{\partial t}+\nabla \cdot[(\rho \psi) \boldsymbol{v}]=\psi \underbrace{\left[\frac{\partial \rho}{\partial t}+\nabla \cdot(\rho \boldsymbol{v})\right]}_{=0}+\rho\left[\frac{\partial \psi}{\partial t}+\boldsymbol{v} \cdot \nabla \psi\right] .
$$

The first bracket is zero according to (63). The second bracket is the material derivative of $\psi$ as derived in section 3.1 .

$$
\frac{\mathrm{D} \psi}{\mathrm{D} t}=\frac{\partial \psi}{\partial t}+\boldsymbol{v} \cdot \nabla \psi
$$

Thus we get the generic balance equation (55) in an equivalent form

$$
\rho \frac{\mathrm{D} \psi}{\mathrm{D} t}=-\nabla \cdot \boldsymbol{\zeta}+\pi
$$

Momentum The momentum equation we get by substituting in (65) $\boldsymbol{\psi}=\boldsymbol{v}, \boldsymbol{\zeta}=-\boldsymbol{S}$ and $\boldsymbol{\pi}=\rho \boldsymbol{g}$ (where $\boldsymbol{\psi}$ and $\boldsymbol{\pi}$ are vectors and $\boldsymbol{\zeta}$ is a second order tensor)

$$
\rho\left[\frac{\partial \boldsymbol{v}}{\partial t}+\boldsymbol{v} \cdot \nabla \boldsymbol{v}\right]=\boldsymbol{\nabla} \cdot \boldsymbol{S}+\rho \boldsymbol{g}
$$

The stress tensor can be divided into a contribution of the fluid at rest and the fluid in motion, $\boldsymbol{S}=-p \boldsymbol{I}+\boldsymbol{T}$. The body force vector is the gravity vector $\boldsymbol{g}$ assuming that there are no other body forces.

If the fluids are Newtonian (linearly viscous) fluids the (deviatoric part of the) stress tensor for both phases is given by $\boldsymbol{T}=\mu\left[\boldsymbol{\nabla} \boldsymbol{v}+(\boldsymbol{\nabla} \boldsymbol{v})^{T}\right]+\frac{\mu^{\prime}}{3}(\boldsymbol{\nabla} \cdot \boldsymbol{v})$, with shear viscosity $\mu$ and modified bulk viscosity $\mu^{\prime}$. Together with the incompressibility condition the momentum equation

$$
\rho\left[\frac{\partial \boldsymbol{v}}{\partial t}+\boldsymbol{v} \cdot \nabla \boldsymbol{v}\right]=-\nabla p+\mu \nabla^{2} \boldsymbol{v}+\rho \boldsymbol{g}
$$

then forms the well known Navier-Stokes equations.

Note that if we had derived the momentum equation from (55), the divergence term in the convective term would be nonlinear. The advantage of deriving the momentum equation from (65) is that the divergence term is then linear which makes numerical discretization easier. 
Energy According to the first law of thermodynamics the increase of internal and kinetic energy in a material control volume is given by heat supply plus power due to work acting on the fluid. The differential equation governing the energy is

$$
\rho \frac{\mathrm{D}}{\mathrm{D} t}\left(e+\frac{v^{2}}{2}\right)=(\boldsymbol{\nabla} \cdot[\boldsymbol{S} \boldsymbol{v}]+\rho \boldsymbol{g} \cdot \boldsymbol{v})+(-\boldsymbol{\nabla} \cdot \boldsymbol{q}+\rho z),
$$

where $e$ is the internal energy per unit mass, $\boldsymbol{S} \boldsymbol{v}$ is the power due to surface forces per unit area, $\rho \boldsymbol{g} \cdot \boldsymbol{v}$ is the gravity power per unit volume, $\boldsymbol{q}$ the heat flux per unit area and $z$ the heat production per unit volume, which is zero in our case. Here $\psi=e+\frac{v^{2}}{2}, \boldsymbol{\zeta}=-\boldsymbol{S} \boldsymbol{v}+\boldsymbol{q}$ and $\pi=\rho \boldsymbol{g} \cdot \boldsymbol{v}+\rho z$. To get the energy in a more commonly used form, we subtract the mechanical energy equation from (67). The mechanical energy equation is formed by the scalar product of momentum equation and velocity. By this we get7

$$
\rho\left[\frac{\partial e}{\partial t}+\boldsymbol{v} \cdot \nabla e\right]=-\boldsymbol{\nabla} \cdot \boldsymbol{q}+\boldsymbol{S} \cdot \boldsymbol{\nabla} \boldsymbol{v} .
$$

Assuming that the heating effect of friction can be neglected, the dissipative term $\boldsymbol{T} \cdot \boldsymbol{\nabla} \boldsymbol{v}$ is zero. Moreover, $\boldsymbol{S} \cdot \boldsymbol{\nabla} \boldsymbol{v}$ vanishes since $\boldsymbol{S} \cdot \boldsymbol{\nabla} \boldsymbol{v}=\boldsymbol{T} \cdot \boldsymbol{\nabla} \boldsymbol{v}-p(\boldsymbol{I} \cdot \boldsymbol{\nabla} \boldsymbol{v})=-p(\boldsymbol{\nabla} \cdot \boldsymbol{v})=0$.

Constitutive equations for internal energy and heat flux complete the equations. For small temperature differences internal energy can be described by a linear function $e=c\left(T-T_{0}\right)+$ $e\left(T_{0}\right)$, where $c$ is the specific heat capacity. The heat flux is given by Fourier's law $\boldsymbol{q}=-\lambda \boldsymbol{\nabla} T$, where $\lambda$ is the heat conductivity. This results in the heat equation

$$
\rho c\left[\frac{\partial T}{\partial t}+\boldsymbol{v} \cdot \nabla T\right]=\lambda \nabla^{2} T .
$$

The material properties viscosity, heat capacity and heat conductivity are in general functions of density, pressure and temperature, but for incompressible fluids only temperature dependency need to be considered. If the temperature interval between the two phases is small the material properties can be assumed to be constant.

\subsection{Mass, momentum and energy jump conditions}

Mass We start with (62). Assuming the bulk variables $\varphi=\rho$ and $\zeta=0$, and the surface variable $\boldsymbol{\zeta}_{S}=\mathbf{0}$, gives for the mass jump condition at the interface

$$
\llbracket \rho[\boldsymbol{v}-\boldsymbol{u}] \cdot \boldsymbol{n} \rrbracket=0 .
$$

Equation (68) states that the amount of mass flux that enters the interface must also leave the interface $\dot{m}_{l}=\dot{m}_{g}$ (so we can omit the index).

\footnotetext{
${ }^{7}$ Here we used the identity $\boldsymbol{\nabla} \cdot(\boldsymbol{S} \boldsymbol{v})=(\boldsymbol{\nabla} \cdot \boldsymbol{S}) \cdot \boldsymbol{v}+\boldsymbol{S} \cdot \boldsymbol{\nabla} \boldsymbol{v}$
} 
Momentum To get the momentum jump condition at the surface between condensate and vapor, we set in (62) $\boldsymbol{\varphi}=\rho \boldsymbol{v}, \boldsymbol{\zeta}=-\boldsymbol{S}$ and $\boldsymbol{\zeta}_{S}=-\boldsymbol{S}_{S}$. As for the stress tensor, the surface stress tensor can be divided into two components $\boldsymbol{S}_{S}=\sigma \boldsymbol{I}_{S}+\boldsymbol{T}_{S}$, where $\boldsymbol{I}_{S}$ is the surface identity tensor (9). Assuming a surface without mass we have $\boldsymbol{T}_{S}=0$ and from the surface stress tensor, only the interfacial tension remains $\boldsymbol{S}_{S}=\sigma \boldsymbol{I}_{S}$, where $\sigma$ is the surface tension coefficient, see section 4.3. Surface tension can be understood as the counterpart of the pressure in the bulk fluid. With this substitution the momentum jump condition becomes

$$
\llbracket \rho \boldsymbol{v}[\boldsymbol{v}-\boldsymbol{u}] \cdot \boldsymbol{n} \rrbracket-\llbracket \boldsymbol{S n} \rrbracket=\nabla_{S} \sigma+2 H \sigma \boldsymbol{n} .
$$

If we neglect temperature dependency of the surface tension coefficient (no Marangony effects) $\sigma$ is constant within the surface.

We split the vector equation (69) into three scalar equations by multiplying it first with the normal vector and then with the two tangential vectors. The tangential equations are equal, so we skip the third equation and use the symbol $\boldsymbol{t}$ to denote both tangential vectors. Further we make use of (68) and the assumption of no-slip at the surface $\llbracket \boldsymbol{v} \cdot \boldsymbol{t} \rrbracket=0$. Then we get

$$
\begin{aligned}
\llbracket \dot{m} \boldsymbol{v} \cdot \boldsymbol{n} \rrbracket+\llbracket p \rrbracket-\llbracket \boldsymbol{n} \cdot \boldsymbol{T} \boldsymbol{n} \rrbracket & =2 H \sigma, \\
\llbracket \boldsymbol{t} \cdot \boldsymbol{T} \boldsymbol{n} \rrbracket & =0 .
\end{aligned}
$$

Energy The energy jump condition is with $\varphi=\rho\left(e+\frac{v^{2}}{2}\right), \boldsymbol{\zeta}=-\boldsymbol{S} \boldsymbol{v}+\boldsymbol{q}, \boldsymbol{\zeta}_{S}=\left(-\sigma \boldsymbol{I}_{S}+\boldsymbol{T}_{S}\right) \boldsymbol{u}=$ $-\sigma \boldsymbol{u}$ and by making the same assumptions as for the momentum jump condition given by

$$
\llbracket \rho\left(e+\frac{v^{2}}{2}\right)[\boldsymbol{v}-\boldsymbol{u}] \cdot \boldsymbol{n} \rrbracket-\llbracket \boldsymbol{n} \cdot \boldsymbol{S} \boldsymbol{v} \rrbracket+\llbracket \boldsymbol{q} \cdot \boldsymbol{n} \rrbracket=\nabla_{S} \cdot(\sigma \boldsymbol{u})+2 H \sigma \boldsymbol{n} \cdot \boldsymbol{u},
$$

where $e$ is the internal energy. Instead of a balance equation for the internal energy a formulation with the enthalpy $h=e+\frac{p}{\rho}$ is more convenient, because enthalpy is a measurable quantity whereas internal energy is not easy to measure.

Writing $\boldsymbol{n} \cdot \boldsymbol{S} \boldsymbol{v}=\boldsymbol{v} \cdot \boldsymbol{S n}(\boldsymbol{S}$ is symmetric), splitting the vectors on the left side of (72) into their normal and tangential components according to $\boldsymbol{a} \cdot \boldsymbol{b}=(\boldsymbol{a} \cdot \boldsymbol{n})(\boldsymbol{b} \cdot \boldsymbol{n})+(\boldsymbol{a} \cdot \boldsymbol{t})(\boldsymbol{b} \cdot \boldsymbol{t})$, and applying the chain rule to $\nabla_{S} \cdot(\sigma \boldsymbol{u})$ gives

$$
\begin{array}{r}
\llbracket \dot{m}\left(e+\frac{(\boldsymbol{v} \cdot \boldsymbol{n})^{2}}{2}+\frac{(\boldsymbol{v} \cdot \boldsymbol{t})^{2}}{2}\right) \rrbracket-\llbracket(\boldsymbol{v} \cdot \boldsymbol{n}) \\
(\boldsymbol{n} \cdot \boldsymbol{S n})+(\boldsymbol{v} \cdot \boldsymbol{t})(\boldsymbol{t} \cdot \boldsymbol{S n}) \rrbracket+\llbracket \boldsymbol{q} \cdot \boldsymbol{n} \rrbracket \\
=\sigma \boldsymbol{\nabla}_{S} \cdot \boldsymbol{u}+\boldsymbol{u} \cdot \nabla_{S} \sigma+2 H \sigma \boldsymbol{n} \cdot \boldsymbol{u} .
\end{array}
$$


Subtracting from the energy jump condition (73) the scalar product of surface velocity $\boldsymbol{u}=$ $(\boldsymbol{u} \cdot \boldsymbol{n}) \boldsymbol{n}$ and momentum jump condition (69)

$$
\llbracket \dot{m}(\boldsymbol{u} \cdot \boldsymbol{n})(\boldsymbol{v} \cdot \boldsymbol{n}) \rrbracket-\llbracket(\boldsymbol{u} \cdot \boldsymbol{n})(\boldsymbol{n} \cdot \boldsymbol{S n}) \rrbracket=\boldsymbol{u} \cdot\left[\nabla_{S} \sigma+2 H \sigma \cdot \boldsymbol{n}\right]
$$

gives together with the no-slip condition and the tangential momentum jump condition (71) after rearranging the pressure term

$$
\begin{array}{r}
\llbracket \dot{m}\left(e+\frac{p}{\rho}+\frac{(\boldsymbol{v} \cdot \boldsymbol{n})^{2}}{2}-(\boldsymbol{u} \cdot \boldsymbol{n})(\boldsymbol{v} \cdot \boldsymbol{n})\right) \rrbracket-\llbracket[\boldsymbol{v}-\boldsymbol{u}] \cdot \boldsymbol{n}(\boldsymbol{n} \cdot \boldsymbol{T} \boldsymbol{n}) \rrbracket+\llbracket \boldsymbol{q} \cdot \boldsymbol{n} \rrbracket \\
=\sigma \boldsymbol{\nabla}_{S} \cdot \boldsymbol{u} .
\end{array}
$$

Using again Chain Rule and that $\boldsymbol{n} \cdot \boldsymbol{\nabla}_{S}=0$ we get for the right hand side

$$
\sigma \nabla_{S} \cdot \boldsymbol{u}=\sigma \boldsymbol{\nabla}_{S} \cdot(\boldsymbol{u} \cdot \boldsymbol{n}) \boldsymbol{n}=\sigma(\boldsymbol{u} \cdot \boldsymbol{n}) \boldsymbol{\nabla}_{S} \cdot \boldsymbol{n}=2 \sigma H \boldsymbol{u} \cdot \boldsymbol{n}
$$

Expanding the kinetic energy term

$$
\llbracket \frac{(\boldsymbol{v} \cdot \boldsymbol{n})^{2}}{2} \rrbracket=\llbracket \frac{(\boldsymbol{v} \cdot \boldsymbol{n}-\boldsymbol{u} \cdot \boldsymbol{n})^{2}}{2}+(\boldsymbol{v} \cdot \boldsymbol{n})(\boldsymbol{u} \cdot \boldsymbol{n})-\frac{(\boldsymbol{u} \cdot \boldsymbol{n})^{2}}{2} \rrbracket
$$

and noting that the surface velocity jump is zero gives the energy jump condition as

$$
\llbracket \dot{m} h \rrbracket+\llbracket \dot{m} \frac{([\boldsymbol{v}-\boldsymbol{u}] \cdot \boldsymbol{n})^{2}}{2} \rrbracket-\llbracket[\boldsymbol{v}-\boldsymbol{u}] \cdot \boldsymbol{n}(\boldsymbol{n} \cdot \boldsymbol{T} \boldsymbol{n}) \rrbracket+\llbracket \boldsymbol{q} \cdot \boldsymbol{n} \rrbracket=2 \sigma H \boldsymbol{u} \cdot \boldsymbol{n} .
$$

If kinetic energy, viscous energy and the effect of surface tension can be neglected, the energy jump condition for an interface between two fluids becomes

$$
\dot{m} \Delta h+\llbracket \boldsymbol{q} \cdot \boldsymbol{n} \rrbracket=0,
$$

where $\Delta h=\llbracket h \rrbracket$ is the latent heat of vaporization.

\subsection{Summary of the model equations}

The two fluids $(i=l, g)$ are described by the continuity equation for an incompressible fluid, the momentum equation and the energy equation:

Continuity equation

const. density

$$
\boldsymbol{\nabla} \cdot \boldsymbol{v}_{i}=0
$$


Momentum equation

$$
\rho_{i}\left(\frac{\partial \boldsymbol{v}_{i}}{\partial t}+\boldsymbol{v}_{i} \cdot \boldsymbol{\nabla} \boldsymbol{v}_{i}\right)=-\underset{\text { inertia }}{\boldsymbol{\nabla} p_{i}}+\underset{\text { pressure }}{\mu_{i} \nabla^{2} \boldsymbol{v}_{i}}+\underset{\text { friction }}{\rho_{i} \boldsymbol{g}}
$$

Energy equation

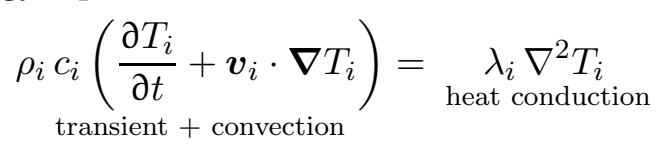

Newtonian fluid

const. viscosity

no dissipation const. heat conductivity const. thermal capacity

The jump conditions at the interface between the two fluids for mass, momentum and energy are with $\llbracket \varphi \rrbracket:=\varphi_{g}-\varphi_{l}$ (dropping the tilde on the normal and tangential vectors):

Mass

$$
\llbracket \rho[\boldsymbol{v}-\boldsymbol{u}] \cdot \boldsymbol{n} \rrbracket=\underset{\text { mass flux }}{\llbracket \dot{m} \rrbracket}
$$

Momentum

const. surface tension coefficient no slip between both phases

$$
\begin{aligned}
& \underset{\text { momentum }}{\llbracket \dot{m} \boldsymbol{v} \cdot \boldsymbol{n} \rrbracket}+\underset{\text { surface pressure }}{\llbracket p p}-\llbracket \boldsymbol{n} \cdot \boldsymbol{T} \cdot \boldsymbol{n} \rrbracket=\underset{\text { surface tension }}{2 H \sigma} \\
& \text { due to } \\
& \text { condensation } \\
& \underset{\text { shear stress }}{\llbracket \boldsymbol{t} \cdot \boldsymbol{T} \cdot \boldsymbol{n} \rrbracket}=0
\end{aligned}
$$

Energy

$$
\underset{\text { condensation }}{\dot{m} \Delta h}=\underset{\text { heat flux }}{\llbracket \boldsymbol{q} \cdot \boldsymbol{n} \rrbracket}
$$

no kinetic energy no dissipation

with $\boldsymbol{T}=\mu\left[\boldsymbol{\nabla} \boldsymbol{v}+(\boldsymbol{\nabla} \boldsymbol{v})^{T}\right]$ and $\boldsymbol{q}=-\lambda \boldsymbol{\nabla} T$. 
Boundary conditions As many boundary conditions for each coordinate of an unknown are necessary as the equation has derivatives of this unknown. One more equation is missing and for this we take the condition of thermodynamic equilibrium at the interface, according to which the fluid temperatures are equal at the moving surface

$$
T_{l}=T_{g}
$$

From the five jump conditions we need one equation to calculate the mass flux, so that four equations remain to calculate the boundary conditions for three velocity components, pressure and temperature. We can use either the mass jump condition or the energy jump condition to calculate the mass flux.

From the remaining equations either the mass jump condition or the normal momentum jump condition can be used to calculate one velocity boundary condition, depending on which equation is used to compute the mass flux. Which of the two equations is used depends on further simplifications of the problem. Often the term with the mass flux in the normal

momentum jump condition is small and can be dropped, then this equation is not available to calculate the mass flux.

The second and third velocity boundary condition are taken from the tangential momentum jump conditions.

\section{SI Units of some variables and material properties}




$\begin{array}{lll}H & \mathrm{~m}^{-1} & \text { mean curvature } \\ \boldsymbol{S} & \mathrm{N} \mathrm{m}^{-2} & \text { stress tensor }\left(\mathrm{J}=\mathrm{kg} \mathrm{m} \mathrm{s}^{-2}\right) \\ \boldsymbol{T} & \mathrm{N} \mathrm{m} & \text { viscous stress tensor } \\ T & \mathrm{~K} & \text { temperature }(\mathrm{C}=\mathrm{K}-273,15) \\ c & \mathrm{~J} \mathrm{~kg}^{-1} \mathrm{~K}^{-1} & \text { specific heat capacity }(\mathrm{J}=\mathrm{N} \mathrm{m}) \\ e & \mathrm{~J} \mathrm{~kg}^{-1} & \text { specific internal energy } \\ \boldsymbol{g} & \mathrm{m} \mathrm{s}^{-2} & \text { gravity vector }\left(g=9,81 \mathrm{~m} \mathrm{~s}^{-2}\right) \\ \alpha & \mathrm{W} \mathrm{m}^{-2} \mathrm{~K}^{-1} & \text { heat transfer coefficient }\left(\mathrm{W}=\mathrm{J} \mathrm{s}^{-2}\right) \\ \Delta h & \mathrm{~J} \mathrm{~kg}^{-1} & \text { latent heat of vaporization } \\ \dot{m} & \mathrm{~kg} \mathrm{~s}^{-1} \mathrm{~m}^{3} & \text { volume specific mass flux } \\ p & \mathrm{~N} \mathrm{~m}^{-2} & \text { pressure } \\ \boldsymbol{q} & \mathrm{J} \mathrm{m}^{-2} \mathrm{~s}^{-1} & \text { heat flux vector } \\ \boldsymbol{u}, \boldsymbol{v} & \mathrm{m} \mathrm{s}^{-1} & \text { velocity } \\ \mu & \mathrm{kg} \mathrm{m}^{-1} \mathrm{~s}^{-1} & \text { dynamical viscosity } \\ \lambda & \mathrm{W} \mathrm{m}^{-1} \mathrm{~K}^{-1} & \text { thermal conductivity } \\ \rho & \mathrm{kg} \mathrm{m}^{-3} & \text { density } \\ \sigma & \mathrm{N} \mathrm{m}^{-1} & \text { surface tension }\end{array}$

\section{References}

[Ap] Tom M. Apostol, Calculus, Wiley, Vol. 2, 2nd ed. 1969.

[Ar] Rutherford Aris, Vectors, Tensors and the Basic Equations of Fluid Mechanics, Dover Publications, 1989.

[BiStLi] R. Byron Bird and Warren E. Stewart and Edwin N. Lightfoot, Transport phenomena, Wiley and Sons, 2002 (1960).

[ChMa] Alexandre J. Chorin and Jerrold E. Marsden, A Mathematical Introduction to Fluid Mechanics, Springer, 1993 (1990, 1979).

[De] William M. Deen, Analysis of Transport Phenomena, Oxford University Press, 1998.

[Ed] David A. Edwards and Howard Brenner and Darsh T. Wasan, Interfacial transport processes and rheology, Butterworth-Heinemann, 1991.

[Er] A. Cemal Eringen, Continuum Physics, Academic Press, Vol. 1, 1971. 
[GaPr] Rene Gatignol and Roger Prud'homme, Mechanical and hermodynamical modeling of fluid interfaces, World Scientific Publishing, 2001.

[Gi] J. Willard Gibbs, Collected Works, Longmanns, Vol. 1, p,218, 1828.

[Hu] Kolumban Hutter and Klaus D. Jöhnk, Continuum Methods of Physical Modeling, Springer, 2005.

[IsHi] Mamrou Ishii and Takashi Hibiki, Thermo-fluid Dynamics of Two-Phase Flow, Springer, 2006.

[Ka] Wilfried Kaplan, Advanced Calculus, Addison-Wesley, 2002 (..., 1993).

[Ko] Nikolay I. Kolev, Multiphase Flow Dynamics 1: Fundamentals, Springer, 2007.

[Kü] Wolfgang Kühnel, Differential Geometry: Curves - Surfaces - Manifolds, American Mathematical Societey, 2005, (2003).

[Op] John Oprea, The mathematics of soap films: Explorations with Maple, American Mathematical Societey, 2000.

[Scha] Heinz Schade and Klaus Neemann, Tensoranalysis, de Gruyter, 2006 (1997).

[Sc] L.E. Scriven, Dynamics of a fluid interface, Chem. Eng. Sci., Vol. 12, p. 98-108, 1960.

[S199] John C. Slattery, Advanced Transport Phenomena, Cambridge University Press, 1999.

[Sl07] John C. Slattery, Interfacial transport phenomena, Springer, 2007 (1990),

[TrTo] Clifford A. Truesdell and Richard Toupin, The classical field theories, in Handbuch der Physik, Bd.III/1, Springer, 1960. 